\title{
$\lambda$-Euler's difference table for colored permutations
}

\author{
Bin $\operatorname{Han}^{*}$ \\ Univ Lyon, Université Claude Bernard Lyon 1 \\ CNRS UMR 5208, Institut Camille Jordan \\ F-69622 Villeurbanne cedex, France \\ han@math.univ-lyon1.fr
}

Submitted: Feb 9, 2018; Accepted: Oct 19, 2018; Published: Nov 2, 2018

(C) The author. Released under the CC BY-ND license (International 4.0).

\begin{abstract}
Motivated by the $\lambda$-Euler's difference table of Eriksen et al. and colored Euler's difference table of Faliharimalala and Zeng, we study the $\lambda$-analogue of colored Euler's difference table and generalize their results. We generalize the number of permutations with $k$-excedances studied by Liese and Remmel in colored permutations. We also extend Wang et al.'s recent results about $r$-derangements by relating with the sequences arising from the difference table.
\end{abstract}

Mathematics Subject Classifications: 05A18, 05A15

\section{Introduction}

Euler [4] studied the difference table $\left(g_{n}^{m}\right)_{0 \leqslant m \leqslant n}$, where the coefficients are defined by $g_{n}^{n}=n !$ and

$$
g_{n}^{m}=g_{n}^{m+1}-g_{n-1}^{m},
$$

for $0 \leqslant m \leqslant n-1$. Dumont and Randrianarivony [4] studied the combinatorial interpretation of $g_{n}^{m}$ in the symmetric group $S_{n}$, which consists of permutations of $[n]=\{1, \ldots, n\}$. In particular, they showed that the sequence $\left\{g_{n}^{0}\right\}_{n \geqslant 0}$ is the number of derangements, i.e., the fixed point free permutations in $S_{n}$. Then Rakotondrajao [11] developed further combinatorial interpretations. The reader is referred to $[4,11,12,7,3,5,10,2]$, where several generalizations of Euler's difference table with combinatorial meanings were studied.

Definition 1. For fixed integer $\ell \geqslant 1$, we define $\lambda$-Euler's difference table $\left(g_{\ell, n}^{m}(\lambda)\right)_{0 \leqslant m \leqslant n}$ for $C_{\ell} \prec S_{n}$, where the coefficients are defined by

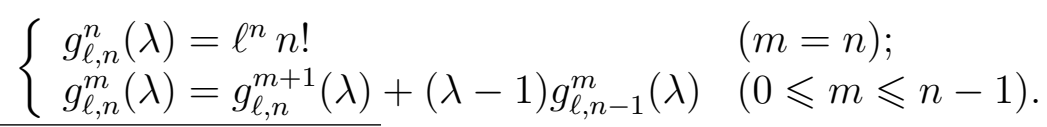

*The author was supported by the China Scholarship Council. 
From the above definition, it is easy to see the coefficients $g_{\ell, n}^{m}(\lambda)$ are polynomials in $\lambda$. Faliharimalala and Zeng [7] studied the combinatorial interpretation of $g_{\ell, n}^{m}(0)$ in terms of $k$-circular successions in $C_{\ell} 2 S_{n}$. Eriksen et al. [5] gave a combinatorial interpretation for the coefficients $g_{1, n}^{m}(\lambda)$ by assuming that $\lambda$ is a non-negative integer. They showed that $g_{1, n}^{m}(\lambda)$ count the number of permutations of $[n]$ such that fixed points on the last $n-k$ positions may be colored in any one of $\lambda$ colors. Liese and Remmel [10] interpreted the coefficients of polynomial $g_{1, n}^{m}(\lambda)$ by counting certain rook placements in the $[n] \times[n]$ board.

It is not hard to see that the coefficient $g_{\ell, n}^{m}(\lambda)$ is divisible by $\ell^{m} m$ !. This prompted us to introduce $d_{\ell, n}^{m}(\lambda)=g_{\ell, n}^{m}(\lambda) / \ell^{m} m$ !. Then we derive the following allied array $\left(d_{\ell, n}^{m}(\lambda)\right)_{0 \leqslant m \leqslant n}$ from (1.2).

Definition 2. For a fixed integer $\ell \geqslant 1$, the coefficients of the $\lambda$-difference table

$$
\left(d_{\ell, n}^{m}(\lambda)\right)_{0 \leqslant m \leqslant n}
$$

are defined by

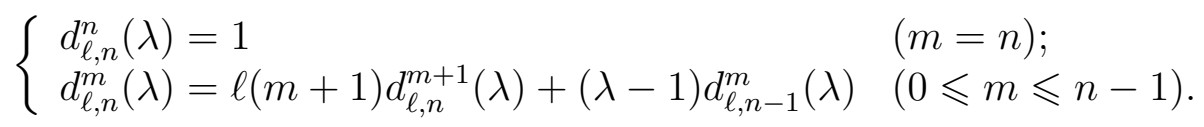

\begin{tabular}{|c|c|c|c|c|}
\hline$n \backslash m$ & 0 & 1 & 2 & 3 \\
\hline 0 & 1 & & & \\
\hline 1 & $\lambda$ & 1 & & \\
\hline 2 & $\lambda^{2}+1$ & $\lambda+1$ & 1 & \\
\hline 3 & $\lambda^{3}+3 \lambda+2$ & $\lambda^{2}+2 \lambda+3$ & $\lambda+2$ & 1 \\
\hline 4 & $\lambda^{4}+6 \lambda^{2}+8 \lambda+9$ & $\lambda^{3}+3 \lambda^{2}+9 \lambda+11$ & $\lambda^{2}+4 \lambda+7$ & $\lambda+3$ \\
\hline
\end{tabular}

The first terms of these coefficients for $\ell=1,2$ are given in Tables 1 and 2 .

\begin{tabular}{|c|c|c|c|c|}
\hline$n \backslash m$ & 0 & 1 & 2 & 3 \\
\hline 0 & 1 & & & \\
\hline 1 & $\lambda+1$ & 1 & & \\
\hline 2 & $\lambda^{2}+2 \lambda+5$ & $\lambda+3$ & 1 & \\
\hline 3 & $\lambda^{3}+3 \lambda^{2}+15 \lambda+29$ & $\lambda^{2}+6 \lambda+17$ & $\lambda+5$ & 1 \\
\hline 4 & $\lambda^{4}+4 \lambda^{3}+30 \lambda^{2}+116 \lambda+233$ & $\lambda^{3}+9 \lambda^{2}+51 \lambda+131$ & $\lambda^{2}+10 \lambda+37$ & $\lambda+7 \quad 1$ \\
\hline
\end{tabular}

Table 1: Values of $d_{\ell, n}^{m}(\lambda)$ for $0 \leqslant m \leqslant n \leqslant 4$ and $\ell=1$.

Table 2: Values of $d_{\ell, n}^{m}(\lambda)$ for $0 \leqslant m \leqslant n \leqslant 4$ and $\ell=2$.

Two combinatorial interpretations of $d_{\ell, n}^{m}(0)$ were given in [7]. When $\lambda$ is a nonnegative integer, Eriksen et al. [5] gave a combinatorial interpretation for the coefficients 
$d_{1, n}^{m}(\lambda)$ in the symmetric group. Wang et al.[14] introduced the $r$-derangement number, which counts the derangements of $[n]$ with the first $r$ elements appear in distinct cycles.

Motivated by $[7,5,10,14]$, we study the combinatorial interpretation of $g_{\ell, n}^{m}(\lambda)$ and $d_{\ell, n}^{m}(\lambda)$ in the colored symmetric group $G_{\ell, n}$, i.e., the wreath product of a cyclic group and a symmetric group. The paper is organized as follows. In Sections 3 and 4, we interpret the polynomial $g_{\ell, n}^{m}(\lambda)$ and the coefficients in $g_{\ell, n}^{m}(\lambda)$, respectively. In Sections 5 and 6 , we prove the linear combinatorial interpretation and cyclic combinatorial interpretation of $d_{\ell, n}^{m}(\lambda)$, respectively. In Section 7 , we obtain the generating functions and recurrence relations of $d_{\ell, n}^{m}(\lambda)$. In Section 8 , we generalize $r$-derangement number by relating with $d_{\ell, n}^{m}(\lambda)$. In Section 9, we give a combinatorial proof of recurrence relation of $d_{\ell, n}^{m}(\lambda)$.

\section{Definitions and main results}

For positive integers $\ell, n \geqslant 1$, the group of colored permutations of $n$ elements with $\ell$ colors is the wreath product $G_{\ell, n}:=C_{\ell}\left(S_{n}=C_{\ell}^{n} \rtimes S_{n}\right.$, where $C_{\ell}$ is the $\ell$-cyclic group generated by $\zeta=e^{2 i \pi / \ell}\left(i^{2}=-1\right)$. From definition, it is obvious to see the elements in $G_{\ell, n}$ are pairs $(\epsilon, \sigma) \in C_{\ell}^{n} \times S_{n}$.

And $G_{\ell, n}$ can also be seen as a permutation group on the colored set:

$$
\Sigma_{\ell, n}:=C_{\ell} \times[n]=\left\{\zeta^{j} i \mid i \in[n], 0 \leqslant j \leqslant \ell-1\right\} .
$$

Clearly there are $\ell^{n} n$ ! signed permutations in the group $G_{\ell, n}$. For more details, see [6].

A signed permutation $\pi \in G_{\ell, n}$ can be written in two-line form. For example, if $\pi=(\epsilon, \sigma) \in G_{4,11}$, where $\epsilon=\left(1, \zeta^{3}, 1, \zeta, 1,1, \zeta^{2}, \zeta, 1, \zeta, 1\right)$ and

$$
\sigma=7 \quad 5 \quad 3 \quad 1 \quad 2 \quad 6 \quad 8 \quad 9 \quad 4 \quad 10 \quad 11,
$$

we write

$$
\pi=\left(\begin{array}{ccccccccccc}
1 & 2 & 3 & 4 & 5 & 6 & 7 & 8 & 9 & 10 & 11 \\
\zeta^{2} 7 & 5 & 3 & 1 & \zeta^{3} 2 & 6 & \zeta 8 & 9 & \zeta 4 & \zeta 10 & 11
\end{array}\right)
$$

To be convenient, we write $j$ bars over $i$ instead of $\zeta^{j} i$. Thus, we rewrite the above permutation in linear form as $\pi=\begin{array}{lllllllllllll}\overline{7} & 5 & 3 & 1 & \overline{2} & 6 & \overline{8} & 9 & \overline{4} & \overline{10} & 11 \text {, or in disjoint cyclic form }\end{array}$ as

$$
\pi=(1, \overline{\overline{7}}, \overline{8}, 9, \overline{4})(\overline{\overline{2}}, 5)(3)(6)(\overline{10})(11) .
$$

That is, when using disjoint cyclic notation to determine the image of a number, we ignore the sign on that number and only consider the sign on the number to which it is mapped. Thus, in the above example, we ignore the sign $\zeta^{2}$ on the 7 and 7 maps to $\zeta 8$ since the sign on 8 is $\zeta$. Moreover, let $[m+1, n]$ denote the interval $\{m+1, \ldots, n\}$, and we give the following conventions:

i) If $\pi=(\epsilon, \sigma) \in G_{\ell, n}$, we define $|\pi|=\sigma$ and $\operatorname{sign}_{\pi}(i)=\epsilon_{i}$ for $i \in[n]$. For example, if $\pi=1 \overline{4} 3 \overline{\overline{2}}$ then $\epsilon=\left(1, \zeta^{2}, 1, \zeta\right)$ and $\operatorname{sign}_{\pi}(4)=\zeta$. 
ii) For $i \in[n]$ and $j \in\{0,1, \ldots, \ell-1\}$, we define $\zeta^{j} i+k=\zeta^{j}(i+k)$ for $0 \leqslant k \leqslant n-i$, and $\zeta^{j} i-k=\zeta^{j}(i-k)$ for $0 \leqslant k \leqslant i$. For example, $\overline{\overline{2}}+1=\overline{\overline{3}}$ in $G_{4,11}$.

iii) We define the total ordering on $\Sigma_{\ell, n}$ as follows. For $i, j \in\{0, \ldots, \ell-1\}$ and $a, b \in[n]$,

$$
\zeta^{i} a<\zeta^{j} b \Longleftrightarrow i>j \quad \text { or } \quad i=j \text { and } a<b \text {. }
$$

In $G_{\ell, n}$, Faliharimalala and Zeng [7] introduced the $k$-successions as follows.

Definition 3. Given a permutation $\pi \in G_{\ell, n}$ and an integer $0 \leqslant k \leqslant n-1, \pi(i)$ is a $k$-succession at position $i \in[n-k]$ if $\pi(i)=i+k$. In particular, the 0 -succession is also called fixed point.

Note that the above $k$-succession $\pi(i)$ needs to be uncolored, that is, $\operatorname{sign}_{\pi}(\pi(i))=1$.

To obtain the combinatorial interpretation of $g_{\ell, n}^{m}(\lambda)$, we introduce the following definition.

Definition 4. For any integer $0 \leqslant k \leqslant n-1$, let $S U C_{k}(\pi)$ denote the set of k-successions in $\pi \in G_{\ell, n}$, i.e.,

$$
S U C_{k}(\pi)=\left\{\pi(i) \mid \pi(i)=i+k, i \in[n-k], \pi \in G_{\ell, n}\right\} .
$$

For an integer $0 \leqslant m \leqslant n$, we define the statistic $\operatorname{suc}_{>m}^{(k)}(\pi)$ as the number of $k$ successions included in $[m+1, n]$ for $\pi \in G_{\ell, n}$, i.e.,

$$
\operatorname{suc}_{>m}^{(k)}(\pi)=\#\left\{\pi(i) \in[m+1, n] \mid \pi(i) \in S U C_{k}(\pi)\right\} .
$$

In particular, for $\pi \in G_{\ell, n}$, by taking $k=0$ and $k=m$, suc ${ }_{>m}^{(k)}$ is the number of fixed points and $m$-successions concerning $\pi \in G_{\ell, n}$, respectively, which are included in $[m+1, n]$.

For example, when $\pi \in G_{4,11}$, if

$$
\pi=531 \overline{\overline{2}} 6 \overline{8} 9 \overline{4} \overline{10} 11 \overline{\overline{7}}
$$

and

$$
\pi^{\prime}=31 \overline{\overline{2}} 6 \overline{8} 9 \overline{4} \overline{10} 11 \overline{\overline{7}} 5,
$$

we have $S U C_{1}(\pi)=S U C_{2}\left(\pi^{\prime}\right)=\{3,6,11\}$ and $\operatorname{suc}_{>4}^{(1)}(\pi)=\operatorname{suc}_{>4}^{(2)}\left(\pi^{\prime}\right)=2$.

Theorem 5. For fixed integers $\ell, k, m$ and $n$, let $\ell \geqslant 1$ and $0 \leqslant k \leqslant m \leqslant n$, we have

$$
g_{\ell, n}^{m}(\lambda)=\sum_{\pi \in G_{\ell, n}} \lambda^{\operatorname{suc}_{>m}^{(k)}(\pi)}
$$

Remark 6. We recover Faliharimalala and Zeng's result [7, Theorem 3] about the combinatorial interpretation of $g_{\ell, n}^{m}(0)$ in $G_{\ell, n}$. And we prove Theorem 5 in Section 3. 
We give an example to illustrate the above theorem. For $\ell=2, n=2$ and $m=1$, the permutations in $G_{2,2}$ are

$$
12, \overline{1} 2,1 \overline{2}, \overline{1} \overline{2}, 21, \overline{2} 1,2 \overline{1}, \overline{2} \overline{1} \text {. }
$$

For $k=0, \sum_{\pi \in G_{2,2}} \lambda^{\mathrm{suc}_{>1}^{(0)}(\pi)}=2 \lambda+6$. For $k=1, \sum_{\pi \in G_{2,2}} \lambda^{\mathrm{suc}_{>1}^{(1)}(\pi)}=2 \lambda+6$.

For $n, m, s \geqslant 0$, Rakotondrajao [12] also studied the number of permutations in $S_{n}$ having exactly $s m$-successions. Similarly, we define that $c_{\ell, n, s}^{m}$ is the number of permutations $\pi \in G_{\ell, n}$ having $s m$-successions. In other words,

$$
c_{\ell, n, s}^{m}=\left|\left\{\pi \in G_{\ell, n}|| S U C_{m}(\pi) \mid=s\right\}\right|, \quad \text { for } n, s, m \geqslant 0 .
$$

With Theorem 5 and above definition, we state an expression of $g_{\ell, n}^{m}(\lambda)$ as follows.

Corollary 7. For $\ell \geqslant 1,0 \leqslant m \leqslant n$ and $0 \leqslant s \leqslant n-m$, we have

$$
g_{\ell, n}^{m}(\lambda)=\sum_{s \geqslant 0} c_{\ell, n, s}^{m} \lambda^{s}
$$

Remark 8. With the equations (2) and (5), we obtain that

$$
c_{\ell, n, s}^{m+1}=c_{\ell, n, s}^{m}+c_{\ell, n-1, s}^{m}-c_{\ell, n-1, s-1}^{m},
$$

which is the result of $[7$, Theorem 4].

To show the combinatorial interpretations and recursions of $c_{\ell, n, s}^{m}$, we review the generalized rook theory model in [1].

Let $B_{n}^{\ell}$ be the $n \times \ell n$ array of squares, we label the $n$ columns from left to right by $1,2, \ldots, n$ and the $\ell n$ rows from bottom to top by

$$
1, \zeta 1, \ldots, \zeta^{\ell-1} 1,2, \zeta 2, \ldots, \zeta^{\ell-1} 2, \ldots, n, \zeta n, \ldots, \zeta^{\ell-1} n,
$$

respectively. For instance, the board $B_{n}^{3}$ is pictured in Figure 1. The square in the column labeled with $i$ and the row labeled with $\zeta^{r} j$ is denoted by $\left(i, \zeta^{r} j\right)$. Each such square is called a cell and the rows labeled by $j, \zeta j, \ldots, \zeta^{\ell-1} j$ are called level $j$.

Given a board $B \subseteq B_{n}^{\ell}$, we let $R_{k, n}^{\ell}(B)$ denote the set of $k$ element subsets $\mathbb{P}$ of $B$ such that no two elements lie in the same level or column for non-negative integers $k$. We call the subset $\mathbb{P}$ a placement of non-attacking $\ell$-rooks in $B$. Since the cells in the placement are considered to contain $\ell$-rooks, we define the $k$ th $\ell$-rook number of $B$ by $r_{k, n}^{\ell}(B)=\left|R_{k, n}^{\ell}(B)\right|$.

Given a permutation $\pi \in G_{\ell, n}$, we can identify $\pi$ with a placement $\mathbb{P}_{\pi}$ of $n \ell$-rooks in $B_{n}^{\ell}$. In other word, $\mathbb{P}_{\pi}=\left\{\left(i, \zeta^{r} j\right): \pi(i)=\zeta^{r} j\right.$ for $\left.1 \leqslant i \leqslant n\right\}$, then we define the $k$ th $\ell$-hit number of B denoted by $h_{k, n}^{\ell}(B)$, which is the number of $\pi \in G_{\ell, n}$ such that the placement $\mathbb{P}_{\pi}$ intersects the board B in exactly $k$ cells, i.e.,

$$
h_{k, n}^{\ell}(B)=\mid\left\{\mathbb{P}_{\pi} \mid \pi \in G_{\ell, n} \text { and }\left|\mathbb{P}_{\pi} \cap B\right|=k\right\} \mid .
$$

Briggs and Remmel [1, Theorem 1] found the following relationship between the $\ell$-hit numbers and the $\ell$-rook numbers. 


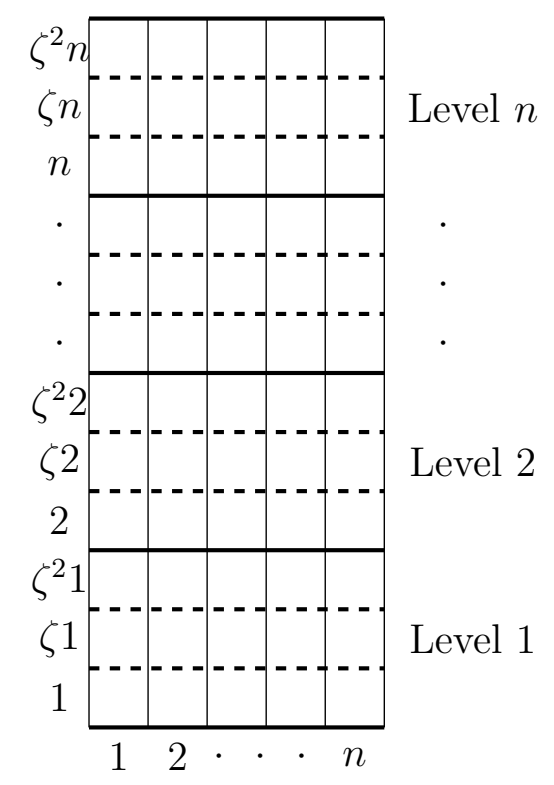

Figure 1: $\mathrm{A}$ board $\mathrm{B}_{n}^{3}$.

Theorem 9 (Briggs-Remmel). Let $B$ be a board contained in $B_{n}^{\ell}$. Then

$$
\sum_{k=0}^{n} h_{k, n}^{\ell}(B) x^{k}=\sum_{k=0}^{n} r_{k, n}^{\ell}(B) \ell^{n-k}(n-k) !(x-1)^{k} .
$$

By interpreting $c_{\ell, n, s}^{m}$ in terms of $\ell$-hit numbers for a certain board, we obtain the following formula.

Theorem 10. For $\ell, n \geqslant 1,0 \leqslant m \leqslant n$ and $s \geqslant 0$, we have

$$
c_{\ell, n, s}^{m}=\sum_{t=s}^{n-m}(-1)^{t-s} \ell^{n-t}(n-t) !\left(\begin{array}{l}
t \\
s
\end{array}\right)\left(\begin{array}{c}
n-m \\
t
\end{array}\right) .
$$

Remark 11. When $\ell=1$, (6) reduce to the result of [10, Theorem 2.2]. And we prove Theorem 10 in Section 4.

To make our arguments of interpreting the coefficients $d_{\ell, n}^{m}(\lambda)$ clear, we only consider the case $k=0$ in statistics $\operatorname{suc}_{>m}^{(k)}(\pi)$. We define $F I X(\pi):=S U C_{0}(\pi)$, which denote the set of fixed points in $\pi \in G_{\ell, n}$, i.e., $F I X(\pi)=\left\{\pi(i) \mid \pi(i)=i, i \in[n], \pi \in G_{\ell, n}\right\}$. Define $\operatorname{fix}_{>m}(\pi):=\operatorname{suc}_{>m}^{(0)}(\pi)$, i.e.,

$$
\operatorname{fix}_{>m}(\pi):=\#\{\pi(i) \in[m+1, n] \mid \pi(i) \in F I X(\pi)\} .
$$

For example, when $\pi \in G_{4,11}$, if $\pi=\overline{\overline{7}} 5331 \overline{\overline{2}} 6 \overline{8} 9 \overline{4} \overline{10} 11$, we have $\operatorname{FIX}(\pi)=$ $\{3,6,11\}$ and $\operatorname{fix}_{>4}(\pi)=2$.

To give the linear interpretation of $d_{\ell, n}^{m}(\lambda)$, we give the following definition. 
Definition 12. For $0 \leqslant m \leqslant n$, a permutation $\pi=(\epsilon, \sigma) \in G_{\ell, n}$ is called an $m$-decreasing permutation if satisfies the following conditions:

i) $\operatorname{sign}_{\pi}(\pi(i))=1(i \in[m])$;

ii) $\pi(1)>\pi(2)>\cdots>\pi(m)$.

Let $L_{\ell, n}^{m}$ be the set of $m$-decreasing permutations in $G_{\ell, n}$. For example, when $\ell=$ $2, n=3$ and $m=2$,

$$
L_{2,3}^{2}=\{213,21 \overline{3}, 312,31 \overline{2}, 321,32 \overline{1}\}, \quad \text { and } \quad \sum_{\pi \in L_{2,3}^{2}} \lambda^{\mathrm{fix}>2(\pi)}=\lambda+5 .
$$

Theorem 13. For $0 \leqslant m \leqslant n$, we have

$$
d_{\ell, n}^{m}(\lambda)=\sum_{\pi \in L_{\ell, n}^{m}} \lambda^{\mathrm{fix}>m}(\pi)
$$

Remark 14 . When $\lambda=0$, Theorem 13 reduce to the result of [7, Theorem 10], we prove above theorem in Section 5.

To give the cyclic interpretation of $d_{\ell, n}^{m}(\lambda)$, we give the following definition.

Definition 15. For $0 \leqslant m \leqslant n$, a permutation $\pi=(\epsilon, \sigma) \in G_{\ell, n}$ is called $m$-separated permutation if satisfies the following conditions:

i) $\operatorname{sign}_{\pi}(i)=1(i \in[m])$;

ii) the first $m$ elements belong into distinct cycles.

Let $C_{\ell, n}^{m}$ be the set of $m$-separated permutations in $G_{\ell, n}$. For example, when $\ell=2, n=$ 3 and $m=2$,

$C_{2,3}^{2}=\{(13)(2),(1 \overline{3})(2),(1)(23),(1)(2 \overline{3}),(1)(2)(3),(1)(2)(\overline{3})\}, \quad$ and $\sum_{\pi \in C_{2,3}^{2}} \lambda^{\mathrm{fix}>2(\pi)}=\lambda+5$.

Theorem 16. For $0 \leqslant m \leqslant n$, we have

$$
d_{\ell, n}^{m}(\lambda)=\sum_{\pi \in C_{\ell, n}^{m}} \lambda^{\mathrm{fix}>m(\pi)} .
$$

Remark 17 . When $\lambda=0$, Theorem 16 reduce to the result of [7, Theorem 12], we prove above theorem in Section 6.

To generalize the definition of $r$-derangement number, we give the following definition.

Definition 18. For $0 \leqslant m \leqslant n$, a permutation $\pi \in G_{\ell, n}$ is called $m$-fixed point-free colored permutation if satisfies the following conditions: 
i) For $i \in[m]$, let $\pi(i) \in[m+1, n]$ and $\operatorname{sign}_{\pi}(i)=\operatorname{sign}_{\pi}(\pi(i))=1$;

ii) no two elements of $[m]$ are in the same cycle.

Let $F_{\ell, n+m}^{m}$ be the set of $m$-fixed point-free colored permutations in $G_{\ell, n+2 m}$, we define

$$
f_{\ell, n+m}^{m}(\lambda)=\sum_{\pi \in F_{\ell, n+m}^{m}} \lambda^{\mathrm{fix}>m(\pi)} .
$$

For example, when $\ell=2, n=1$ and $m=1$,

$$
F_{2,2}^{1}=\{(12)(3),(12)(\overline{3}),(13)(2),(13)(\overline{2}),(123),(12 \overline{3}),(132),(13 \overline{2})\}
$$

and $f_{2,2}^{1}(\lambda)=2 \lambda+6$.

Remark 19. When $(\ell, \lambda)=(1,0)$, the equation $(7)$ reduce to the sum over

$$
\left\{\pi \in F_{\ell, n+m}^{m} \mid \operatorname{fix}_{>m}(\pi)=0\right\}
$$

then the polynomial $f_{\ell, n+m}^{m}(\lambda)$ reduces to the $r$-derangement number, see [14, Definition 1 ]. By the above definition, we generalize the generating functions and recurrence relations of Wang et al. [14].

By observing the above definitions, we prove the following combinatorial relation between the $f_{\ell, n+m}^{m}(\lambda)$ and $d_{\ell, n+m}^{m}(\lambda)$ in Section 8 .

Theorem 20. For $\ell \geqslant 1$ and $m, n \geqslant 0$, we have

$$
f_{\ell, n+m}^{m}(\lambda)=\frac{(n+m) !}{n !} d_{\ell, n+m}^{m}(\lambda)
$$

\section{Proof of Theorem 5}

In the section, to prove Theorem 5 , we prove the following equations,

$$
\left\{\begin{aligned}
\sum_{\pi \in G_{\ell, n}} \lambda^{\operatorname{suc}_{>n}^{(k)}(\pi)}=\ell^{n} n ! & & (m=n) ; \\
\sum_{\pi \in G_{\ell, n}} \lambda^{\operatorname{suc}_{>m}^{(k)}(\pi)}=\sum_{\pi \in G_{\ell, n}} \lambda^{\operatorname{suc}_{>m+1}^{(k)}(\pi)}+(\lambda-1) & \sum_{\pi \in G_{\ell, n-1}} \lambda^{\operatorname{suc}_{>m}^{(k)}(\pi)} & (0 \leqslant m \leqslant n-1) .
\end{aligned}\right.
$$

Lemma 21. For any integer $k$ such that $0 \leqslant k \leqslant m$ and $0 \leqslant m \leqslant n$, there holds

$$
\sum_{\substack{\pi \in G_{\ell, n} \\ m+1 \in S U C_{k}(\pi)}} \lambda^{\operatorname{suc}_{>m}^{(k)}(\pi)}=\lambda \sum_{\pi \in G_{\ell, n-1}} \lambda^{\operatorname{suc}_{>m}^{(k)}(\pi)} .
$$


Proof. Let us define the bijection $\psi: G_{\ell, n} \mapsto G_{\ell, n-1}$, For $\pi \in G_{\ell, n}$, we delete the $m+1$ at position $m+1-k$ and define the $\psi(\pi)=\widehat{\pi}_{1} \widehat{\pi}_{2} \ldots \widehat{\pi}_{m-k} \widehat{\pi}_{m-k+2} \ldots \widehat{\pi}_{n-1} \in G_{\ell, n-1}$ where

$$
\widehat{\pi}_{i}=\left\{\begin{array}{rll}
\pi_{i}, & \text { if } & |\pi|_{i}<m+1 \\
\pi_{i}-1, & \text { if } & |\pi|_{i}>m+1 .
\end{array}\right.
$$

Conversely, starting from $\psi(\pi)=\widehat{\pi}_{1} \widehat{\pi}_{2} \ldots \widehat{\pi}_{m-k} \widehat{\pi}_{m-k+2} \ldots \widehat{\pi}_{n} \in G_{\ell, n-1}$, we define $\pi=$ $\pi_{1} \pi_{2} \ldots \pi_{n} \in G_{\ell, n}$ where

$$
\pi_{i}=\left\{\begin{aligned}
\widehat{\pi}_{i}, & \text { if } \quad|\widehat{\pi}|_{i}<m+1 \\
\widehat{\pi}_{i}+1, & \text { if } \quad|\widehat{\pi}|_{i} \geqslant m+1 .
\end{aligned}\right.
$$

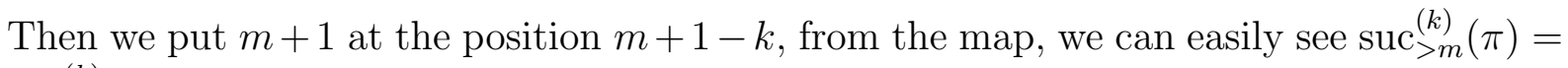
$\operatorname{suc}_{>m}^{(k)}(\psi(\pi))+1$.

For example $\ell=4, n=9, m=4, k=1, \pi=\overline{\overline{7}} 345 \overline{\overline{2}} \overline{1} 89 \overline{6}, \psi(\pi)=\overline{\overline{6}} 34 \overline{\overline{2}} \overline{1} 78 \overline{5}$, and $\operatorname{suc}_{>4}^{(1)}(\pi)=\operatorname{suc}_{>4}^{(1)}(\psi(\pi))+1$.

Lemma 22. For $0 \leqslant m \leqslant n$, there holds

$$
\sum_{\pi \in G_{\ell, n-1}} \lambda^{\operatorname{suc}_{>m}^{(k)}(\pi)}=\sum_{\substack{\pi \in G_{\ell, n} \\ m+1 \in S U C_{k}(\pi)}} \lambda^{\operatorname{suc}_{>m+1}^{(k)}(\pi)}
$$

Proof. It follows similar arguments as in the proof of Lemma 21.

Proof of Theorem 5. First we check the initial condition in (9), when $m=n$, $\operatorname{suc}_{>n}^{(k)}(\pi)=$ $0, \sum_{\pi \in G_{\ell, n}} \lambda^{\text {suc }_{>n}^{(k)}(\pi)}=\ell^{n} n !$

We start to prove the recurrence in (9). Then, by considering the following equation,

$$
\sum_{\pi \in G_{\ell, n}} \lambda^{\mathrm{suc}_{>m}^{(k)}(\pi)}=\sum_{\substack{\pi \in G_{\ell, n} \\ m+1 \notin S U C_{k}(\pi)}} \lambda^{\operatorname{suc}_{>m}^{(k)}(\pi)}+\sum_{\substack{\pi \in G_{\ell, n} \\ m+1 \in S U C_{k}(\pi)}} \lambda^{\operatorname{suc}_{>m}^{(k)}(\pi)} .
$$

Because for $\pi \in G_{\ell, n}$ with $m+1 \notin S U C_{k}(\pi)$, we have $\operatorname{suc}_{>m}^{(k)}(\pi)=\operatorname{suc}_{>m+1}^{(k)}(\pi)$, then (12) is equivalent to

$$
\sum_{\pi \in G_{\ell, n}} \lambda^{\operatorname{suc}_{>m}^{(k)}(\pi)}=\sum_{\substack{\pi \in G_{\ell, n} \\ m+1 \notin S U C_{k}(\pi)}} \lambda^{\operatorname{suc}_{>m+1}^{(k)}(\pi)}+\sum_{\substack{\pi \in G_{\ell, n} \\ m+1 \in S U C_{k}(\pi)}} \lambda^{\operatorname{suc}_{>m}^{(k)}(\pi)}
$$

By equations (10) and (13), we obtain that

$$
\sum_{\pi \in G_{\ell, n}} \lambda^{\mathrm{suc}_{>m}^{(k)}(\pi)}=\sum_{\substack{\pi \in G_{\ell, n} \\ m+1 \notin S U C_{k}(\pi)}} \lambda^{\operatorname{suc}_{>m+1}^{(k)}(\pi)}+\lambda \sum_{\pi \in G_{\ell, n-1}} \lambda^{\mathrm{suc}_{>m}^{(k)}(\pi)} .
$$




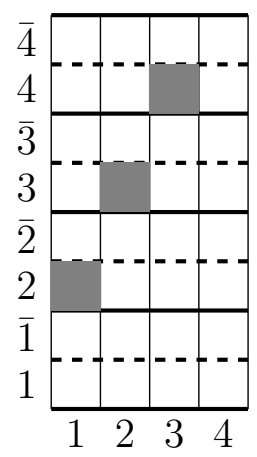

Figure 2: The board $\mathrm{B}_{4,1}^{2}$ corresponds to the shaded cells.

By combining the equations (11) and (14), we obtain

$$
\sum_{\pi \in G_{\ell, n}} \lambda^{\mathrm{suc}_{>m}^{(k)}(\pi)}=(\lambda-1) \sum_{\pi \in G_{\ell, n-1}} \lambda^{\mathrm{suc}_{>m}^{(k)}(\pi)}+\sum_{\substack{\pi \in G_{\ell, n} \\ m+1 \notin S U C_{k}(\pi)}} \lambda^{\mathrm{suc}_{>m+1}^{(k)}(\pi)}+\sum_{\substack{\pi \in G_{\ell, n} \\ m+1 \in S U C_{k}(\pi)}} \lambda^{\mathrm{suc}_{>m+1}^{(k)}(\pi)} .
$$

With (12), it is easy to see that the above equation is equivalent to the recurrence relation in (9), this completes the proof of Theorem 5 .

Remark 23. Since why $g_{\ell, n}^{m}(\lambda)$ is independent from $k(0 \leqslant k \leqslant m)$ in the above proof is not mentioned, we state an argument as follows. By considering the bijection $d$ which transforms $\pi=\pi_{1} \pi_{2} \pi_{3} \cdots \pi_{n}$ into $d(\pi)=\pi^{\prime}=\pi_{2} \pi_{3} \cdots \pi_{n} \pi_{1}$. It is easy to see that the $k$-successions of $\pi$ are in $[m+1, n]$ if and only if the $(k+1)$-successions of $\pi^{\prime}$ are in $[m+1, n]$. Hence, let the composition of $j$ times of $d$ is denoted by $d^{j}$, the application of $d^{k_{2}-k_{1}}$ permits to transfer the $k_{1}$-successions to $k_{2}$-successions if $k_{1}<k_{2}$. In particular if we apply $d^{m}$ to a permutation whose fixed points are in $[m+1, n]$, then we obtain a permutation whose $m$-succession are in $[m+1, n]$ and vice versa.

\section{Proof of Theorem 10}

In this section, first we prove the following expressions of $c_{\ell, n, s}^{m}$ in Theorem 10,

$$
c_{\ell, n, s}^{m}=\sum_{t=s}^{n-m}(-1)^{t-s} \ell^{n-t}(n-t) !\left(\begin{array}{l}
t \\
s
\end{array}\right)\left(\begin{array}{c}
n-m \\
t
\end{array}\right) .
$$

Then we derive several recurrence relations of $c_{\ell, n, s}^{m}$.

Proof of Theorem 10. First, we give the combinatorial interpretation of $c_{\ell, n, s}^{m}$ as follows. Let $B_{n, m}^{\ell}$ be the board contained in $B_{n}^{\ell}$ consisting of the cells $(1,1+m),(2,2+m)$, $(3,3+m), \ldots,(n-m, n)$. For example, the board $B_{4,1}^{2}$ is pictured in Figure 2 . Then the number of $\pi \in G_{\ell, n}$ with $s m$-successions is the $s$-th $\ell$-hit number of $B_{n, m}^{\ell}$, i.e.,

$$
c_{\ell, n, s}^{m}=h_{s, n}^{\ell}\left(B_{n, m}^{\ell}\right)
$$


With the definitions of $B_{n, m}^{\ell}$, we have $r_{s, n}^{\ell}\left(B_{n, m}^{\ell}\right)=\left(\begin{array}{c}n-m \\ s\end{array}\right)$. By Theorem 9,

$$
\begin{aligned}
g_{\ell, n}^{m}(\lambda) & =\sum_{s=0}^{n} c_{\ell, n, s}^{m} \lambda^{s}=\sum_{s=0}^{n} h_{s, n}^{\ell}\left(B_{n, m}^{\ell}\right) \lambda^{s} \\
& =\sum_{s=0}^{n} r_{s, n}^{\ell}\left(B_{n, m}^{\ell}\right) \ell^{n-s}(n-s) !(\lambda-1)^{s} \\
& =\sum_{s=0}^{n}\left(\begin{array}{c}
n-m \\
s
\end{array}\right) \ell^{n-s}(n-s) !(\lambda-1)^{s} .
\end{aligned}
$$

Equating the coefficients of $\lambda^{s}$ yields (15) immediately.

Remark 24. We also obtain the above expression (17) of $g_{\ell, n}^{m}(\lambda)$ by generating function, see Proposition 41.

Let $s=n-m$ in (15), we obtain the following corollary.

Corollary 25. For all $\ell \geqslant 1$ and $n \geqslant m \geqslant 0$,

$$
c_{\ell, n, n-m}^{m}=\ell^{m} m !
$$

Next we show the recurrence relations of $c_{\ell, n, s}^{m}$ in colored symmetric group.

Proposition 26. For all $\ell \geqslant 1, n \geqslant 2,0 \leqslant m<n$, and $s \geqslant 1$,

$$
c_{\ell, n, s}^{m}=(\ell(n-s-1)+(\ell-1)) c_{\ell, n-1, s}^{m}+\ell(s+1) c_{\ell, n-1, s+1}^{m}+c_{\ell, n-1, s-1}^{m} .
$$

Proof. Let us consider the map from $\pi=\pi_{1} \ldots \pi_{n-1} \in G_{\ell, n-1}$ to $\bar{\pi} \in G_{\ell, n}$ such that $\bar{\pi}$ has $s m$-successions, we consider the following three cases.

- If $\pi \in G_{\ell, n-1}$ has $s m$-successions.

1. Let $\bar{\pi}=\pi_{1} \pi_{n-m-1}\left(\zeta^{j} n\right) \pi_{n-m+1} \ldots \pi_{n-1} \pi_{n-m}$ and $1 \leqslant j \leqslant \ell-1$, the number of $m$-successions of $\bar{\pi}$ and $\pi$ are the same, so there are $(\ell-1) c_{\ell, n-1, s}^{m}$ permutations in this case.

2. Let $\bar{\pi}=\pi_{1} \pi_{i-1}\left(\zeta^{j} n\right) \pi_{i+1} \ldots \pi_{n-1} \pi_{i}$, where $i \neq n-m$ and $i$ is a position without $m$-successions, the number of $m$-successions of $\bar{\pi}$ and $\pi$ are the same. Since we have $n-s-1$ choices for position $i$ and $0 \leqslant j \leqslant \ell-1$, there are $\ell(n-s-1) c_{\ell, n-1, s}^{m}$ permutations in this case.

- If $\pi \in G_{\ell, n-1}$ has $s+1 m$-successions. Let $\bar{\pi}=\pi_{1} \pi_{i-1}\left(\zeta^{j} n\right) \pi_{i+1} \ldots \pi_{n-1} \pi_{i}$, where $i$ is a position with $m$-succession, the number of $m$-successions of $\bar{\pi}$ is the number of $m$-successions of $\pi$ minus one. Since we have $s+1$ choices for position $i$ and $0 \leqslant j \leqslant \ell-1$, there are $\ell(s+1) c_{\ell, n-1, s+1}^{m}$ permutations in this case. 


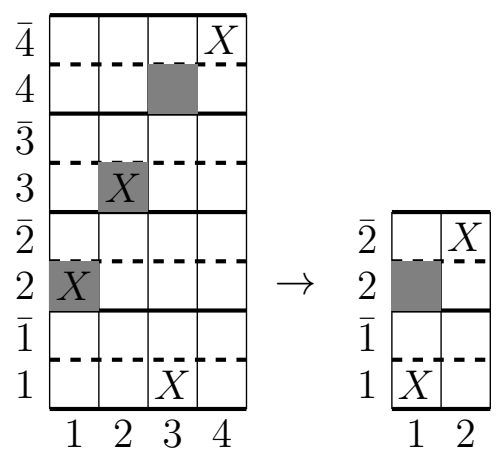

Figure 3: From Board $B_{4}^{2}$ to Board $B_{2}^{2}$.

- If $\pi \in G_{\ell, n-1}$ has $s-1 m$-successions. Let $\bar{\pi}=\pi_{1} \pi_{n-m-1}\left(\zeta^{j} n\right) \pi_{n-m+1} \ldots \pi_{n-1} \pi_{n-m}$ and $j=0$, the number of $m$-successions of $\bar{\pi}$ is the number of $m$-successions of $\pi$ plus one, so there are $c_{\ell, n-1, s-1}^{m}$ permutations in this case.

Proposition 27. For all $\ell, n \geqslant 1,0 \leqslant m<n$, and $s \geqslant 0$,

$$
c_{\ell, n, s}^{m}=\left(\begin{array}{c}
n-m \\
s
\end{array}\right) c_{\ell, n-s, 0}^{m}
$$

Proof. Note that in (16), $c_{\ell, n, s}^{m}$ is the number of placements of $n$ non-attacking $\ell$-rooks in $B_{n}^{\ell}$ that intersect $B_{n, m}^{\ell}$ in exactly $s$ squares. By removing the level $i+m$ and column $i$ of these $\ell$-rooks which lie in the cell $(i, i+m)(1 \leqslant i \leqslant n-m)$, we obtain these placements of $n-s$ non-attacking $\ell$-rooks in $B_{n-s}^{\ell}$ that intersect $B_{n-s, m}^{\ell}$ in exactly 0 squares, which is counted by $c_{\ell, n-s, 0}^{m}$. The process is pictured in Figure 3.

Remark 28. Faliharimalala and Zeng [7, Lemma 14] proved the above (19) directly by interpreting $c_{\ell, n, s}^{m}$ as the number of permutation in $G_{\ell, n}$ with s $m$-successions. However, we give a trivial proof by interpreting $c_{\ell, n, s}^{m}$ as the number of placements of $n$ non-attacking $\ell$-rooks in $B_{n}^{\ell}$ that intersect $B_{n, m}^{\ell}$ in exactly $s$ squares.

Proposition 29. For all $\ell \geqslant 1, n \geqslant 2$ and $0 \leqslant m<n$,

$$
c_{\ell, n, 0}^{m}=(\ell n-1) c_{\ell, n-1,0}^{m}+\ell(n-m-1) c_{\ell, n-2,0}^{m} .
$$

Proof. Let us consider the map from $\pi=\pi_{1} \ldots \pi_{n} \in G_{\ell, n}$ to $\pi^{\prime} \in G_{\ell, n-1}$, starting from $\pi$ without $m$-successions, we define

$$
\pi^{\prime}= \begin{cases}\pi_{1} \ldots \pi_{i-1} \pi_{n} \pi_{i+1} \ldots \pi_{n-1}, & \text { if } \pi_{i}=\zeta^{j} n(0 \leqslant j \leqslant \ell-1) \text { for } 1 \leqslant i<n \\ \pi_{1} \ldots \pi_{n-1}, & \text { if } \pi_{n}=\zeta^{j} n(0 \leqslant j \leqslant \ell-1) .\end{cases}
$$


1. $\pi^{\prime} \in G_{\ell, n-1}$ has no $m$-successions.

Either $\pi_{n}=\zeta^{j} n(0 \leqslant j \leqslant \ell-1)$ or $\pi_{i}=\zeta^{j} n(1 \leqslant i<n, 0 \leqslant j \leqslant \ell-1)$ and $\pi_{n} \neq i+m, \pi^{\prime}$ has no $m$-successions. Conversely, for $\pi^{\prime} \in G_{\ell, n-1}$ without $m$ successions, by inserting $\zeta^{j} n(0 \leqslant j \leqslant \ell-1)$ into $\pi^{\prime}$ in every position except putting $n$ in to position $n-m$, we obtain the permutation in $G_{\ell, n}$ without $m$-successions. Since $\zeta^{j} n(0 \leqslant j \leqslant \ell-1)$ can be in any position except $\pi_{n-m}=n$, there are $(\ell n-1) c_{n-1,0}^{m}$ permutations.

2. $\pi^{\prime} \in G_{\ell, n-1}$ has $1 m$-succession.

When $\pi_{i}=\zeta^{j} n(1 \leqslant i \leqslant n-1-m, 0 \leqslant j \leqslant \ell-1)$ and $\pi_{n}=i+m, \pi^{\prime}$ has 1 $m$-succession, then the $1 m$-succession of $\pi^{\prime}$ corresponds to the $\ell$-rook $(i, i+m)$ of the rook placement in the board $B_{n-1}^{\ell}$. For the rook placement corresponds to $\pi^{\prime}$ in $B_{n-1}^{\ell}$, removing the column $i$ and level $i+m$ from the board $B_{n-1}^{\ell}$, we obtain the rook placement in $B_{n-2}^{\ell}$ without intersecting $B_{n-2, m}^{\ell}$, which corresponding to the permutation denoted by $\tilde{\pi} \in G_{\ell, n-2}$, and $\widetilde{\pi}$ has no $m$-successions.

Conversely, let $\tilde{\pi}$ be a permutation in $G_{\ell, n-2}$ without $m$-successions, we obtain $\pi \in G_{\ell, n}$ in two steps.

Step 1. For $1 \leqslant i \leqslant n-1-m$, by adding the column $i$ and level $i+m$ to the boards $B_{n-2}^{\ell}$, we choose $(i, i+m)$ as the new $\ell$-rook and take the same rook placement corresponds to $\tilde{\pi}$, then we obtain the rook placement in $B_{n-1}^{\ell}$ corresponding to $\pi^{\prime} \in G_{\ell, n-1}$ with $1 m$-succession.

Step 2. Adding the column $n$ and level $n$ in the board $B_{n-1}^{\ell}$, by taking away the $\ell$ rook $(i, i+m)$ and putting $\ell$-rooks at $\left(i, \zeta^{j} n\right)(0 \leqslant j \leqslant \ell-1)$ and $(n, i+m)$, we obtain the rook placement without intersecting $B_{n, m}^{\ell}$, which corresponds to the permutation $\pi \in G_{\ell, n}$ without $m$-successions. Since $1 \leqslant i \leqslant n-1-m$ and $0 \leqslant j \leqslant \ell-1$, there are $\ell(n-1-m) c_{\ell, n-2,0}^{m}$ permutations.

Remark 30. When $m=0$, we define that

$$
D_{n}^{\ell}:=c_{\ell, n, 0}^{0},
$$

which counts the number of derangements in $G_{\ell, n}$. It is easy to see (20) reduce to

$$
D_{n}^{\ell}=(\ell n-1) D_{n-1}^{\ell}+\ell(n-1) D_{n-2}^{\ell} .
$$

Proposition 31. For all $\ell \geqslant 1, n \geqslant 2$, and $1 \leqslant m<n$,

$$
c_{\ell, n, 0}^{m}=\ell m c_{\ell, n-1,0}^{m-1}+\ell(n-m) c_{\ell, n-1,0}^{m} .
$$

Proof. We prove the above equation by considering level 1 in the rook placement corresponding to the permutation $\pi \in G_{\ell, n}$ without $m$-successions. 


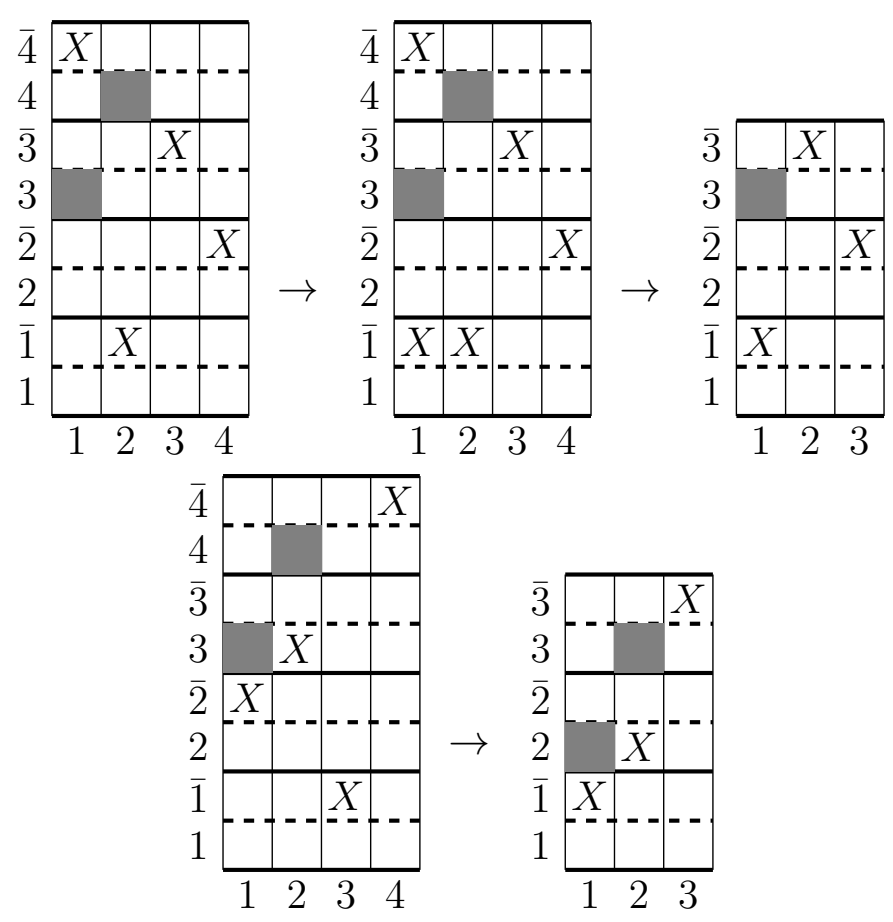

Figure 4: Reducing rook placements from $B_{4,2}^{2}$ by the rook position in level 1.

1. When the $\ell$-rook of level 1 is in column $i(1 \leqslant i \leqslant n-m)$, if the $\ell$-rook of level $i+m$ lies at the position $\left(k, \zeta^{j}(i+m)\right)(k \neq i$ and $0 \leqslant j \leqslant \ell-1)$, by adding a $\ell$-rook at the position $\left(k, \zeta^{j} 1\right)$ in the level 1 , we obtain a placement of $n+1 \ell$-rooks without intersecting $B_{n, m}^{\ell}$. Then removing column $i$ and level $i+m$ will result in a placement of $n-1$ non-attacking $\ell$-rooks without intersecting $B_{n-1, m}^{\ell}$. Since the rook placement corresponding to $\pi$ has $\ell$ different positions in level 1 and column $1 \leqslant i \leqslant n-m$, thus there are $\ell(n-m) c_{\ell, n-1,0}^{m}$ placements. The process is illustrated in top of the Figure 4.

2. When the $\ell$-rook of level 1 is in column $i(n-m<i \leqslant n)$, by removing column $i$ and level 1 , we obtain a placement of $n-1$ non-attacking $\ell$-rooks without intersecting $B_{n-1, m-1}^{\ell}$. Thus there are $\ell m c_{\ell, n-1,0}^{m-1}$ placements. The process is illustrated in the bottom of Figure 4.

Proposition 32. For all $\ell, n \geqslant 1$ and $0 \leqslant m<n$,

$$
c_{\ell, n, 0}^{m}=\ell^{m} m ! \sum_{r=0}^{m}\left(\begin{array}{c}
m \\
r
\end{array}\right)\left(\begin{array}{c}
n-m \\
m-r
\end{array}\right) c_{\ell, n-m, 0}^{m-r} .
$$

Proof. To obtain a placement of $n$ non-attacking $\ell$-rooks without intersecting $B_{n, m}^{\ell}$, starting from the lightly shaded cells in the lower right corner of the board in Figure 5, we see that 0 to $m$-rooks can be placed in this area. Suppose that we choose $r$ levels in 


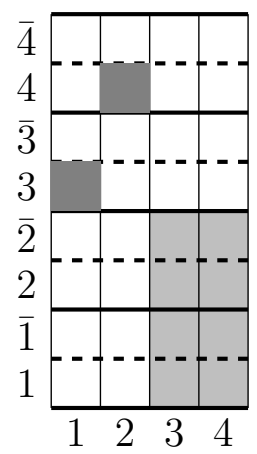

Figure 5: Board $\mathrm{B}_{4,2}^{2}$ with some lightly shaded cells.

this area, there are $\left(\begin{array}{c}m \\ r\end{array}\right)$ ways. Since there should be $m$-rooks in the last $m$ columns, we choose $m-r \ell$-rooks above the lower right corner of the board. Thus we choose $m-r$ levels from the $n-m$ levels, there are $\left(\begin{array}{c}n-m \\ m-r\end{array}\right)$ ways.

After picking the $m$ levels that contain the $\ell$-rooks in the last $m$ columns, there are $\ell^{m} m$ ! ways to place the $\ell$-rooks in the last $m$ columns.

Let $P$ denote the non-attacking rook placement in the last $m$ columns with $r \ell$-rooks falling in the lightly shaded area, we extend $P$ to a non-attacking rook placement $Q$ with $n \ell$-rooks, where there is no intersection with $B_{n, m}^{\ell}$. By removing the levels and columns of rook placement $P$, we obtain the non-attacking rook placement without intersecting $B_{n-m, m-r}^{\ell}$, which is counted by $c_{\ell, n-m, 0}^{m-r}$. Summing over all possible values of $r$ yields the desired result.

Proposition 33. For all $\ell \geqslant 1, n \geqslant 2$ and $0 \leqslant m<n$,

$$
c_{\ell, n, 0}^{m}=\ell c_{\ell, n, 1}^{m+1}+(\ell m+\ell-1) c_{\ell, n-1,0}^{m} .
$$

Proof. Let us consider the rook position of level $n$ in the rook placement which corresponding to the permutation $\pi \in G_{\ell, n}$ without $m$-successions.

1. When the $\ell$-rook of level $n$ is in column $i(1 \leqslant i \leqslant n-m-1)$. If the $\ell$-rook of column $i$ is in row $n$, we keep it unchanged. If the $\ell$-rook of column $i$ is in row $\zeta^{j} n(1 \leqslant j \leqslant \ell-1)$, we exchange the row $\zeta^{j} n$ with row $n$. Then we move the level $n$ to the bottom level of the board, which is denoted by level $1^{\prime}$, other levels are increased by one such as level $2^{\prime}, \ldots$, level $n^{\prime}$. By exchanging the level $1^{\prime}$ and level $(i+m+1)^{\prime}$, we obtain a non-attacking rook placement that intersect $B_{n, m+1}^{\ell}$ one rook $(i, i+m+1)$. Since the $\ell$-rook can be in the row $\zeta^{j} n(0 \leqslant j \leqslant \ell-1)$, there are $\ell c_{n, 1}^{m+1}$ permutations in this case. This process is shown in top of Figure 6 .

2. When the $\ell$-rook of level $n$ is in column $i(n-m \leqslant i \leqslant n)$, the $\ell$-rook can be in the position $\left(i, \zeta^{j} n\right)(n-m \leqslant i \leqslant n, 0 \leqslant j \leqslant \ell-1)$, since $\pi$ has no $m$-successions, the $\ell$-rook can not be in the square $(n-m, n)$, so there are $\ell m+\ell-1$ choices in level $n$. 


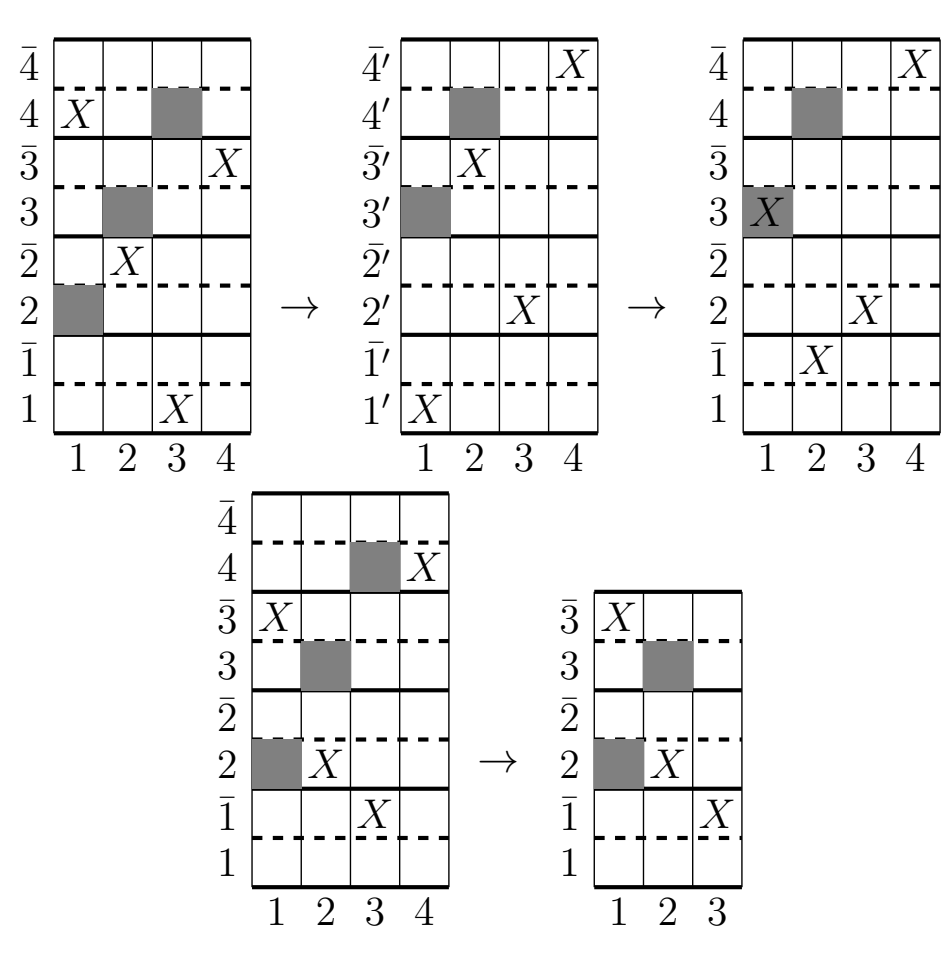

Figure 6: Reducing rook placements from $B_{4,1}^{2}$ by the rook position in top level.

Removing the level $n$ and column $i$ will result in a non-attacking rook placement without intersecting $B_{n-1, m}^{\ell}$. Then there are $(\ell m+\ell-1) B_{n-1, m}^{\ell}$ permutations in this kind. This process is shown in bottom of Figure 6 .

Proposition 34. For $\ell \geqslant 1, n \geqslant 2$ and $1 \leqslant m<n$,

$$
c_{\ell, n, 0}^{m}=c_{\ell, n, 0}^{m-1}+c_{\ell, n-1,0}^{m-1} .
$$

Proof. Let us consider the non-attacking rook placement corresponding to the permutation $\pi \in G_{\ell, n}$ without $m$-successions. We move the bottom level to the top level and all other levels reduced by one, which is shown in Figure 7.

1. When the bottom $\ell$-rook is not in the position $(n-m+1,1)$, the process is shown in the top of Figure 7. After the movement of $\ell$-rooks in the board, we obtain the non-attacking rook placement without intersecting $B_{n, m-1}^{\ell}$. Thus there are $c_{\ell, n, 0}^{m-1}$ permutations in this case.

2. When the bottom $\ell$-rook is in the position $(n-m+1,1)$, the process is shown in the bottom of Figure 7 . The resulting rook placement intersect $B_{n, m-1}^{\ell}$ in the position $(n-m+1, n)$. By removing the column $n-m+1$ and level $n$, we get the non-attacking rook placement without intersecting $B_{n-1, m-1}^{\ell}$. Thus there are $c_{\ell, n-1,0}^{m-1}$ permutations in this case. 


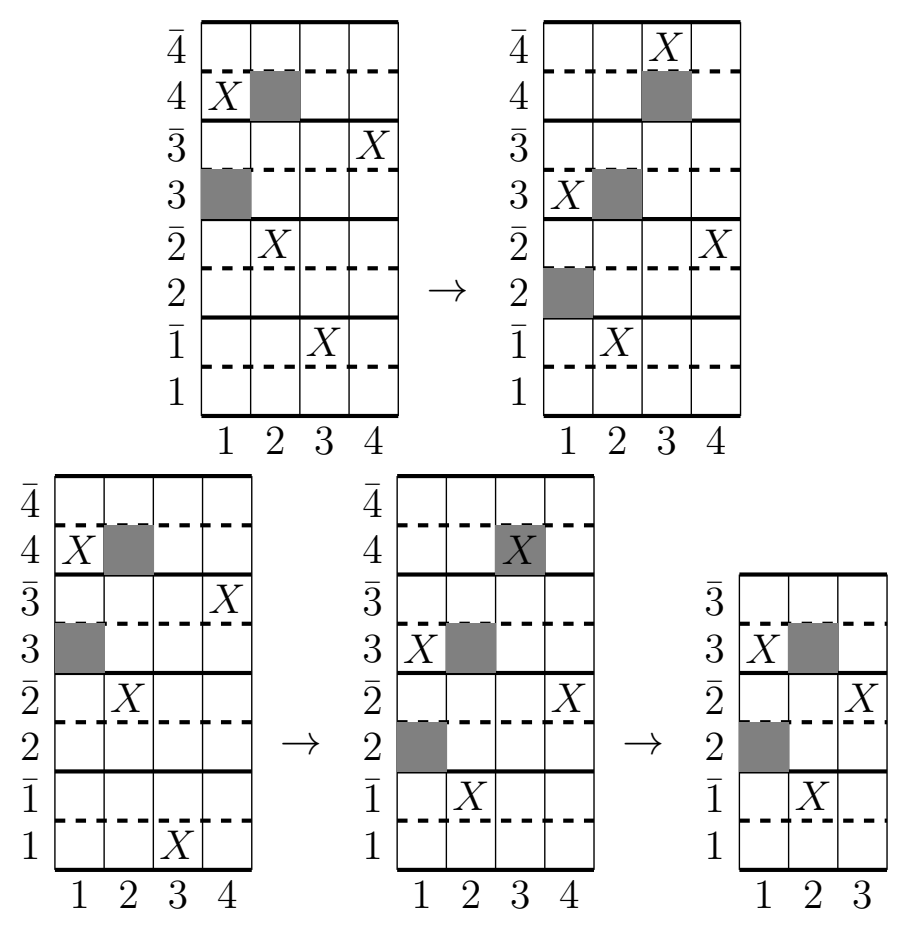

Figure 7: Moving the bottom level to top level in $B_{4,2}^{2}$.

Proposition 35. For $\ell, n \geqslant 1$ and $0 \leqslant m<n$,

$$
c_{\ell, n, 0}^{m}=\sum_{r=0}^{m}\left(\begin{array}{c}
m \\
r
\end{array}\right) D_{n-m+r}^{\ell} .
$$

Proof. We prove this theorem by inductions on $m$. If $m=0$, we have $c_{\ell, n, 0}^{0}=D_{n}^{\ell}$ by equation (21). Suppose that $c_{\ell, n, 0}^{i}=\sum_{r=0}^{i}\left(\begin{array}{l}i \\ r\end{array}\right) D_{n-i+r}^{\ell}$ is satisfied for $i \leqslant m-1$, then

$$
c_{\ell, n, 0}^{m-1}+c_{\ell, n-1,0}^{m-1}=\sum_{r=0}^{m-1}\left(\begin{array}{c}
m-1 \\
r
\end{array}\right) D_{n-m+1+r}^{\ell}+\sum_{r=0}^{m-1}\left(\begin{array}{c}
m-1 \\
r
\end{array}\right) D_{n-m+r}^{\ell} .
$$

By separating out the $m-1$ term of the first sum and the 0 term of the second sum in (27), which is equivalent to

$$
\left(\begin{array}{c}
m-1 \\
m-1
\end{array}\right) D_{n}^{\ell}+\sum_{r=0}^{m-2}\left(\begin{array}{c}
m-1 \\
r
\end{array}\right) D_{n-m+1+r}^{\ell}+\left(\begin{array}{c}
m-1 \\
0
\end{array}\right) D_{n-m}^{\ell}+\sum_{r=1}^{m-1}\left(\begin{array}{c}
m-1 \\
r
\end{array}\right) D_{n-m+r}^{\ell} .
$$

By transforming $r$ to $r-1$ in the first sum and using $\left(\begin{array}{c}m-1 \\ r-1\end{array}\right)+\left(\begin{array}{c}m-1 \\ r\end{array}\right)=\left(\begin{array}{c}m \\ r\end{array}\right)$, we have

$$
c_{\ell, n, 0}^{m-1}+c_{\ell, n-1,0}^{m-1}=\sum_{r=0}^{m}\left(\begin{array}{c}
m \\
r
\end{array}\right) D_{n-m+r}^{\ell} .
$$


With the recurrence $(25)$,

$$
c_{\ell, n, 0}^{m}=c_{\ell, n, 0}^{m-1}+c_{\ell, n-1,0}^{m-1},
$$

we obtain

$$
c_{\ell, n, 0}^{m}=\sum_{r=0}^{m}\left(\begin{array}{c}
m \\
r
\end{array}\right) D_{n-m+r}^{\ell} .
$$

The proof is thus completed.

With equations (19) and (26), we give the relation between $c_{\ell, n, s}^{m}$ and $D_{n}^{\ell}$ directly.

Corollary 36. For $\ell, n \geqslant 1,0 \leqslant m<n$ and $s \geqslant 0$,

$$
c_{\ell, n, s}^{m}=\left(\begin{array}{c}
n-m \\
s
\end{array}\right) \sum_{r=0}^{m}\left(\begin{array}{c}
m \\
r
\end{array}\right) D_{n-s-m+r}^{\ell} .
$$

By observing the coefficients of polynomial $g_{\ell, n}^{m}(\lambda)$, we find $c_{\ell, n, s}^{m}$ decreases as $s$ increases.

Proposition 37. For $\ell, n \geqslant 1,1 \leqslant m<n$ and $s \geqslant 1$,

$$
c_{\ell, n, s-1}^{m} \geqslant c_{\ell, n, s}^{m}
$$

Proof. With recursion (19) and (22), we have

$$
\begin{aligned}
& c_{\ell, n, s-1}^{m}-c_{\ell, n, s}^{m} \\
= & \left(\begin{array}{c}
n-m \\
s-1
\end{array}\right) c_{\ell, n-s+1,0}^{m}-\left(\begin{array}{c}
n-m \\
s
\end{array}\right) c_{\ell, n-s, 0}^{m} \\
= & \left(\ell(n-m-s+1)\left(\begin{array}{c}
n-m \\
s-1
\end{array}\right)-\left(\begin{array}{c}
n-m \\
s
\end{array}\right)\right) c_{\ell, n-s, 0}^{m}+\ell m\left(\begin{array}{c}
n-m \\
s-1
\end{array}\right) c_{\ell, n-s, 0}^{m-1} .
\end{aligned}
$$

Since

$$
\ell(n-m-s+1)\left(\begin{array}{c}
n-m \\
s-1
\end{array}\right)-\left(\begin{array}{c}
n-m \\
s
\end{array}\right)=\frac{(n-m) !}{(n-m-s) ! s !}(\ell s-1),
$$

we obtain (28) immediately.

Remark 38. In particular, when $m=0$, we have the similar result for $c_{\ell, n, s}^{0}$. By using $D_{n}^{\ell}=\ell n D_{n-1}^{\ell}+(-1)^{n}\left[7\right.$, equation (2.8)] and similar arguments above, we have $c_{\ell, n, s-1}^{0} \geqslant$ $c_{\ell, n, s}^{0}$ for $2 \leqslant s \leqslant n-2$ and $n \geqslant 3$.

Remark 39. When $\ell=1$, the above expressions and relations of $c_{\ell, n, s}^{m}$ in this section reduce to Liese and Remmel's results [10, Sections 2 and 3]. 


\section{Proof of Theorem 13}

In this section, to prove Theorem 13, we prove the following equations,

$$
\sum_{\pi \in L_{\ell, n}^{m}} \lambda^{\mathrm{fix}>m(\pi)}=\sum_{\pi \in G_{\ell, n}} \lambda^{\mathrm{fix}>m(\pi)} / \ell^{m} m !, \text { for } 0 \leqslant m \leqslant n .
$$

Proof of Theorem 13. For $1 \leqslant k \leqslant n$, if $\pi=\pi(1) \pi(2) \ldots \pi(k-1) \pi(k) \pi(k+1) \ldots \pi(n) \in$ $G_{\ell, n}$, let $T(\pi)$ be the vector that record the numbers of the last $n-k$ positions in $\pi$, i.e., $T(\pi)=(\pi(k+1), \pi(k+2), \ldots, \pi(n))$. For example, if $n=12, k=4, \pi=$ $\overline{9} 5 \overline{\overline{4}} 13 \overline{\overline{8}} 26 \overline{7} 10 \overline{12} 11 \in G_{4,12}$, then $T(\pi)=(3, \overline{\overline{8}}, \ldots, 11)$. We define the relation $\sim$ on $G_{\ell, n}$ by

$$
\pi \sim \pi^{\prime} \Leftrightarrow T(\pi)=T\left(\pi^{\prime}\right)
$$

it is easy to see this is an equivalence relation. Let us consider the map $\delta:(\eta, \pi) \rightarrow \delta(\eta, \pi)$ from $G_{\ell, m} \times G_{\ell, n}$ to $G_{\ell, n}$, where $G_{\ell, m}$ can be seen as a permutation group of colored set $C_{\ell} \times\{|\pi|(1),|\pi|(2), \ldots,|\pi|(m)\}$. Define the permutation $\delta(\eta, \pi)$ such that $\delta(\eta, \pi)(i)=$ $\eta(i)(i \leqslant m)$, and $\delta(\eta, \pi)(i)=\pi(i)(i>m)$. For example, if $\pi=\overline{9} 5 \overline{\overline{4}} 13 \overline{\overline{8}} 26 \overline{7} 10 \overline{12} 11 \epsilon$ $G_{4,12}$, and $\eta=5 \overline{4} 1 \overline{9} \in G_{4,4^{\prime}}$, then

$$
\delta(\eta, \pi)=5 \overline{4} 1 \overline{9} 3 \overline{\overline{8}} 26 \overline{7} 10 \overline{12} 11 .
$$

So the equivalence class of $\pi \in G_{\ell, n}$ is $\left\{\delta(\eta, \pi) \mid \eta \in G_{\ell, m}\right\}$, it's easy to see the cardinality of each equivalence class is $\ell^{m} m$ !, choosing the representative of the equivalence class $\delta(\iota, \pi)$ such that

$$
\operatorname{sign}(|\iota|(i))=1 \quad \text { and } \quad \iota(1)>\iota(2) \cdots>\iota(m) .
$$

Since the fix points of $\pi$ and $\delta(\iota, \pi)$ on $[m+1, n]$ keep unaltered. By Theorem 5 , we obtain that the number of equivalence class is $g_{\ell, n}^{m}(\lambda) / \ell^{m} m$ !, which yields the equation (29).

Remark 40. As in the proof of Theorem 5, we can also prove the recurrence relations of (3) by constructing bijections directly, the proof is left to the interested reader.

\section{Proof of Theorem 16}

In this section, we give two proofs of Theorem 16. In the first proof, we give a bijection from $C_{\ell, n}^{m}$ to $L_{\ell, n}^{m}$, that is,

$$
\sum_{\pi \in L_{\ell, n}^{m}} \lambda^{\mathrm{fix}>m}(\pi)=\sum_{\pi \in C_{\ell, n}^{m}} \lambda^{\mathrm{fix}_{>m}(\pi)} \text { for } 0 \leqslant m \leqslant n .
$$

In the second proof, we prove this cyclic result by constructing a equivalence relation on $G_{\ell, n}$, that is,

$$
\sum_{\pi \in C_{\ell, n}^{m}} \lambda^{\mathrm{fix}>m(\pi)}=\sum_{\pi \in G_{\ell, n}} \lambda^{\mathrm{fix}>m(\pi)} / \ell^{m} m !, \text { for } 0 \leqslant m \leqslant n .
$$




\subsection{First Proof}

we will give a bijection $\rho: \pi \rightarrow \pi^{\prime}$ from $C_{\ell, n}^{m}$ to $L_{\ell, n}^{m}$ such that fix $\operatorname{sim}_{>}(\pi)=\operatorname{fix}_{>m}\left(\pi^{\prime}\right)$. First we give the map $|\pi| \rightarrow^{\prime}\left|\pi^{\prime}\right|$ and then construct the sign transformation.

- Let $\left|\pi^{\prime}\right|=\left|\pi^{\prime}\right|(1), \ldots,\left|\pi^{\prime}\right|(m), \ldots,\left|\pi^{\prime}(n)\right|$, where $\left|\pi^{\prime}\right|(1), \ldots,\left|\pi^{\prime}\right|(m)$ in decreasing rearrangement of $|\pi|(1), \ldots,|\pi|(m)$ and $\left|\pi^{\prime}\right|(m+i)=|\pi|(m+i)(1 \leqslant i \leqslant n-m)$. Conversely, we give the reverse map by $\pi^{\prime} \rightarrow \pi$ from $L_{\ell, n}^{m}$ to $C_{\ell, n}^{m}$. For $\pi^{\prime} \in L_{\ell, n}^{m}$, we define $P:=\left\{\left|\pi^{\prime}\right|(i), i \in[m]\right\}$. let

$$
\left(\left|\pi^{\prime}\right|^{-s}(i), \ldots,\left|\pi^{\prime}\right|^{-2}(i),\left|\pi^{\prime}\right|^{-1}(i), i\right)
$$

be the cycle of $|\pi|$ containing $i(i \in[m])$, where $s$ is the least non-negative number such that $\left|\pi^{\prime}\right|^{-s}(i) \in P$ and if $\left|\pi^{\prime}\right|(j)=i(j \in[n])$, then $\left|\pi^{\prime}\right|^{-1}(i):=j$. And setting $\left|\pi^{\prime}\right|^{0}(i)=i$, that is, if $i \in P \cap[m]$, then $s=0$, and $i$ is a fixed point of $|\pi|$. The other cycles keep in accordance with $\left|\pi^{\prime}\right|$.

- We define the sign transformation as follows. Since each element $i \in[m]$ in $\pi$ and $\pi(i)(i \in[m])$ in $\pi^{\prime}$ are uncolored, we exchange the sign of $|\pi|(i) \in[m]$ in $\pi$ and $i \in[m]$ in $\pi^{\prime}$. In other words,

$$
\operatorname{sign}_{\pi}(i)=\operatorname{sign}_{\pi^{\prime}}(|\pi|(i))=1 \quad \text { and } \quad \operatorname{sign}_{\pi}(|\pi|(i))=\operatorname{sign}_{\pi^{\prime}}(i), \quad i \in[m] .
$$

The signs of other elements remain unchanged, i.e.,

$$
\operatorname{sign}_{\pi^{\prime}}(i)=\operatorname{sign}_{\pi}(i), \quad i \notin[m] \cup\{|\pi|(i) \mid i \in[m]\} .
$$
$C_{4,12}^{4}$,

For example: For $\ell=4, n=12, m=4, \pi=(1 \overline{\overline{9}})(2 \overline{7})(35)(4)(6 \overline{8})(10)(11 \overline{\overline{12}}) \in$

$$
\operatorname{sign}_{\pi^{\prime}}(1)=\operatorname{sign}_{\pi}(|\pi|(1))=\zeta^{2}, \operatorname{sign}_{\pi^{\prime}}(2)=\operatorname{sign}_{\pi}(|\pi|(2))=\zeta
$$

we have

$$
\pi^{\prime}=97543 \overline{8} \overline{2} 6 \overline{\overline{1}} 10 \overline{\overline{12}} 11 \in L_{4,12}^{4} \quad \text { and } \quad \operatorname{fix}_{>4}(\pi)=\operatorname{fix}_{>4}\left(\pi^{\prime}\right)=1 .
$$

\subsection{Second Proof}

We decompose a permutation $\pi \in G_{\ell, n}$ as a product of disjoint cycles. For each $i \in[m]$, we define $\omega_{\pi}(i)=\pi(i) \pi^{2}(i) \ldots \pi^{s-1}(i)$ where $s \geqslant 1$ is the least integer such that $|\pi|^{s}(i) \in[m]$. Obviously $\omega_{\pi}(i)=\varnothing$ if $s=1$. Let $\Omega(\pi)$ be the product of cycles of $\pi$ which have no common elements with $\left\{\zeta^{j} i \mid i \in[m], 0 \leqslant j \leqslant \ell-1\right\}$, let $\pi_{m} \in G_{\ell, m}$ be the permutation obtained from $\pi$ by deleting elements in $\omega_{\pi}(i)$ and the cycles in $\Omega(\pi)$ for $i \in[m]$.

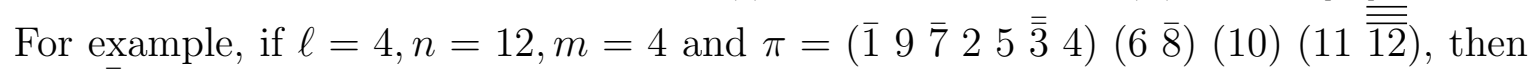
$\pi_{4}=(\overline{1} 2 \overline{\overline{3}} 4)$ and

$$
\omega_{\pi}(1)=9 \overline{7}, \quad \omega_{\pi}(2)=5, \quad \omega_{\pi}(3)=\varnothing, \quad \omega_{\pi}(4)=\varnothing, \quad \text { and } \quad \Omega(\pi)=(6 \overline{8})(10)(11 \overline{\overline{12}}) .
$$


Setting $E(\pi)=\left(\omega_{\pi}(1), \omega_{\pi}(2), \cdots, \omega_{\pi}(k), \Omega(\pi)\right)$, we define the relation $\sim$ on $G_{\ell, n}$ by

$$
\pi_{1} \sim \pi_{2} \Leftrightarrow E\left(\pi_{1}\right)=E\left(\pi_{2}\right)
$$

it is easy to see that this is an equivalence relation. Then we define the mapping $\theta$ : $(\tau, \pi) \mapsto \theta(\tau, \pi)$ from $G_{\ell, m} \times G_{\ell, n}$ to $G_{\ell, n}$. We obtain the permutation $\theta(\tau, \pi)$ by inserting the elements $\omega_{i}(\pi)$ after the elements $\zeta^{j} i(i \in[m], 0 \leqslant j \leqslant \ell-1)$ of $\tau$ and adding the cycles of $\Omega(\pi)$.

For example, if $\pi=(\overline{1} 9 \overline{7} 25 \overline{\overline{3}} 4)(6 \overline{8})(10)(11 \overline{\overline{12}})$ and $\tau=(1 \overline{2})(\overline{3})(4)$ then

$$
\theta(\tau, \pi)=(19 \overline{7} \overline{2} 5)(\overline{3})(4)(6 \overline{8})(10)(11 \overline{\overline{12}}) .
$$

Obviously $\left\{\theta(\tau, \pi) \mid \tau \in G_{\ell, m}\right\}$ is the equivalence class of $\pi \in G_{\ell, n}$. From the construction of $\theta(\tau, \pi)$, for $\tau \in G_{\ell, m}$ and $\pi \in G_{\ell, n}^{m}$, we have $\theta(\tau, \pi) \sim \pi$. Conversely, if $\pi^{\prime} \sim \pi$, then $\pi^{\prime}=\theta\left(\pi_{m}^{\prime}, \pi\right)$, and if $\theta(\tau, \pi)=\theta\left(\tau^{\prime}, \pi\right)=\pi^{\prime}$ for $\tau, \tau^{\prime} \in G_{\ell, m}$, then $\tau=\tau^{\prime}=\pi_{m}^{\prime}$. Hence the cardinality of each equivalence class is $\ell^{m} m$ !. Let $\eta$ be the identity permutation of $G_{\ell, m}$, then we choose $\theta(\eta, \pi)$ as the representative of each equivalence class $\left\{\theta(\tau, \pi) \mid \tau \in G_{\ell, m}\right\}$, that is, $\theta(\eta, \pi)$ represents the the permutation $\pi \in G_{\ell, n}$ where $\operatorname{sign}_{\pi}(i)=1(i \in[m])$ with the first $m$ elements belong into distinct cycles. It is obvious to see $\operatorname{fix}_{>m}(\pi)=\operatorname{fix}_{>m}(\theta(\eta, \pi))$. By Theorem 5, the number of equivalence classes is $g_{\ell, n}^{m}(\lambda) / \ell^{m} m$ !, as desired.

\section{Generating functions and further recurrence relations}

In this section, by using the recurrence relation (2), we obtain the generating functions and further recurrence relations of $g_{\ell, n}^{m}(\lambda)$ and $d_{\ell, n}^{m}(\lambda)$.

Proposition 41. For $m \geqslant 0$ we have the following identities:

$$
\begin{gathered}
g_{\ell, n+m}^{m}(\lambda)=\sum_{i=0}^{n}(\lambda-1)^{n-i}\left(\begin{array}{c}
n \\
i
\end{array}\right) \ell^{m+i}(m+i) !, \\
\sum_{n \geqslant 0} g_{\ell, n+m}^{m}(\lambda) \frac{u^{n}}{n !}=\frac{\ell^{m} m ! \exp ((\lambda-1) u)}{(1-\ell u)^{m+1}} \\
\sum_{m, n \geqslant 0} g_{\ell, n+m}^{m}(\lambda) \frac{x^{m}}{m !} \frac{u^{n}}{n !}=\frac{\exp ((\lambda-1) u)}{1-\ell x-\ell u} .
\end{gathered}
$$

Proof. For any function $f(k)(k \geqslant 0): \mathbb{Z}[\lambda] \rightarrow \mathbb{C}[\lambda]$, we define the operator $\Delta f(n)(\lambda)=$ $f(n)(\lambda)+(\lambda-1) f(n-1)(\lambda)$. By inductions on $N \geqslant 0$, we have

$$
\Delta^{N} f(n)(\lambda)=\sum_{i=0}^{N}(\lambda-1)^{i}\left(\begin{array}{c}
N \\
i
\end{array}\right) f(n-i)(\lambda)=\sum_{i=0}^{N}(\lambda-1)^{N-i}\left(\begin{array}{c}
N \\
i
\end{array}\right) f(n-N+i)(\lambda) .
$$


If $f(n)(\lambda)=g_{\ell, n}^{n}(\lambda)$, thus $g_{\ell, n+m}^{n+m-i}(\lambda)=\Delta^{i} f(n+m)(\lambda)$ for $i \geqslant 0$. From (35), we obtain

$$
g_{\ell, n+m}^{m}(\lambda)=\Delta^{n} f(n+m)(\lambda)=\sum_{i=0}^{n}(\lambda-1)^{n-i}\left(\begin{array}{c}
n \\
i
\end{array}\right) \ell^{m+i}(m+i) ! .
$$

For the above identity, multiplying both sides by $u^{n} / n$ ! and summing over $n \geqslant 0$, we obtain

$$
\sum_{n \geqslant 0} g_{\ell, n+m}^{m}(\lambda) \frac{u^{n}}{n !}=\ell^{m} m ! \sum_{n, i \geqslant 0}(\lambda-1)^{n-i}\left(\begin{array}{c}
m+i \\
i
\end{array}\right) \frac{\ell^{i} u^{n}}{(n-i) !} .
$$

By shifting $n$ to $n+i$, we have

$$
\sum_{n \geqslant 0} g_{\ell, n+m}^{m}(\lambda) \frac{u^{n}}{n !}=\ell^{m} m !\left(\sum_{n \geqslant 0}(\lambda-1)^{n} \frac{u^{n}}{n !}\right) \cdot\left(\sum_{i \geqslant 0}\left(\begin{array}{c}
m+i \\
i
\end{array}\right)(\ell u)^{i}\right) .
$$

Clearly the above equation implies (33) immediately. Finally multiplying both sides of (33) by $x^{m} / m$ ! and summing over $m \geqslant 0$ yields (34).

Remark 42. Setting $m=0$ in (32), we obtain

$$
d_{\ell, n}^{0}(\lambda)=g_{\ell, n}^{0}(\lambda)=n ! \sum_{i=0}^{n} \frac{(\lambda-1)^{i}}{i !} \ell^{n-i},
$$

which implies immediately the following recurrence relation,

$$
d_{\ell, n}^{0}(\lambda)=\ln d_{\ell, n-1}^{0}(\lambda)+(\lambda-1)^{n} \quad(n \geqslant 1) .
$$

Proposition 43. For $\ell \geqslant 1$ and $0 \leqslant m \leqslant n-2$ we have

$$
\begin{aligned}
& g_{\ell, n}^{m}(\lambda)=(\ell n+\lambda-1) g_{\ell, n-1}^{m}(\lambda)-\ell(n-m-1)(\lambda-1) g_{\ell, n-2}^{m}(\lambda) \quad(n \geqslant 2) ; \\
& g_{\ell, n}^{m}(\lambda)=\ell(n-m) g_{\ell, n-1}^{m}(\lambda)+\ell m g_{\ell, n-1}^{m-1}(\lambda) \quad(m \geqslant 1, n \geqslant 1) ; \\
& g_{\ell, n}^{m}(\lambda)=\ell n g_{\ell, n-1}^{m}(\lambda)+\ell m(\lambda-1) g_{\ell, n-2}^{m-1}(\lambda) \quad(m \geqslant 1, n \geqslant 2),
\end{aligned}
$$

where $g_{\ell, 0}^{0}(\lambda)=1, g_{\ell, 1}^{0}(\lambda)=\lambda+\ell-1$ and $g_{\ell, 1}^{1}(\lambda)=\ell$.

Proof. Let $F(u)$ denote the left-hand side of (33). By using the differentiation of $F(u)$ and (33), we obtain

$$
(1-\ell u) F^{\prime}(u)=[\ell(m+1)+(\lambda-1)(1-\ell u)] F(u) .
$$

By equating the coefficients of $u^{n} / n$ ! in (42), we have

$$
g_{\ell, n+m+1}^{m}(\lambda)=[\ell(m+n+1)+\lambda-1] g_{\ell, n+m}^{m}(\lambda)-\ell n(\lambda-1) g_{\ell, n+m-1}^{m}(\lambda),
$$

shifting $n+m+1$ to $n$ yields (39) immediately. 
Then multiplying both sides of (33) by $1-\ell u$, we have

$$
(1-\ell u) \sum_{n \geqslant 0} g_{\ell, n+m}^{m}(\lambda) \frac{u^{n}}{n !}=\frac{\ell^{m} m ! \exp ((\lambda-1) u)}{(1-\ell u)^{m}}=\ell m \sum_{n \geqslant 0} g_{\ell, n+m-1}^{m-1}(\lambda) \frac{u^{n}}{n !} .
$$

By equating the coefficients of $u^{n} / n$ !, we have

$$
g_{\ell, n+m}^{m}(\lambda)-\ell n g_{\ell, n+m-1}^{m}(\lambda)=\ell m g_{\ell, n+m-1}^{m-1}(\lambda),
$$

shifting $n+m$ to $n$ yields (40).

Finally, from (40) and (2), we have

$$
\begin{aligned}
g_{\ell, n}^{m}(\lambda) & =\ell n g_{\ell, n-1}^{m}(\lambda)-\ell m\left(g_{\ell, n-1}^{m}(\lambda)-g_{\ell, n-1}^{m-1}(\lambda)\right) \\
& =\ell n g_{\ell, n-1}^{m}(\lambda)+\ell m(\lambda-1) g_{\ell, n-2}^{m-1}(\lambda),
\end{aligned}
$$

which yields (41), the proof is completed.

With the above Proposition 43, we derive the following propositions immediately.

Proposition 44. For $\ell \geqslant 1$ and $0 \leqslant m \leqslant n-2$ we have

$$
\begin{aligned}
& d_{\ell, n}^{m}(\lambda)=(\ell n+\lambda-1) d_{\ell, n-1}^{m}(\lambda)-\ell(n-m-1)(\lambda-1) d_{\ell, n-2}^{m}(\lambda) \quad(n \geqslant 2) ; \\
& d_{\ell, n}^{m}(\lambda)=\ell(n-m) d_{\ell, n-1}^{m}(\lambda)+d_{\ell, n-1}^{m-1}(\lambda) \quad(m \geqslant 1, n \geqslant 1) ; \\
& d_{\ell, n}^{m}(\lambda)-(\lambda-1) d_{\ell, n-2}^{m-1}(\lambda)=\ell n d_{\ell, n-1}^{m}(\lambda) \quad(m \geqslant 1, n \geqslant 2),
\end{aligned}
$$

where $d_{\ell, 0}^{0}(\lambda)=1, d_{\ell, 1}^{0}(\lambda)=\lambda+\ell-1$ and $d_{\ell, 1}^{1}(\lambda)=1$.

Proof. With Proposition 43, we can get these equations (45), (46) and (47) directly.

Remark 45. Setting $\ell=1,(3)$, (45), and (47) reduce to the result of Eriksen et al. [5, Propositions 8.1, 8.3 and 8.2]. In this case, (33) and (34) recover the result of Rakotondrajao [11, Theorem 6.7 and Theorem 6.8]. Setting $\lambda=0$, Propositions 41, 43 and 44 reduce to the result of Faliharimalala and Zeng [7, Propositions 17, 18, and 19].

\section{Proof of Theorem 20}

In this section, to prove Theorem 20, we prove the following equation,

$$
\sum_{\pi \in F_{\ell, n+m}^{m}} \lambda^{\mathrm{fix}>m(\pi)}=\frac{(n+m) !}{n !} \sum_{\pi \in C_{\ell, n+m}^{m}} \lambda^{\mathrm{fix}>m(\pi)} \text { for } m, n \geqslant 0 .
$$

And with the generating functions of $d_{\ell, n}^{m}(\lambda)$, we obtain the generating functions and recurrence relations of $f_{\ell, n}^{m}(\lambda)$. 
Proof of Theorem 20. For $\ell \geqslant 1$, we construct such a permutation $\pi \in F_{\ell, n+m}^{m}$ in following way, see Definition 18.

Starting from the set $[n+2 m]$, we take $m$ elements from the set $[m+1,2 m]$ as the image of $[1, m]$, which is labeled as $\pi(i)(i \in[m])$. Clearly there are $\left(\begin{array}{c}n+m \\ m\end{array}\right) m$ ! ways to choose. Let $i^{\prime}(i \in[m])$ represent the two element set $\{i, \pi(i)\}$, and let $i^{\prime}(i \in[m+1, n+m])$ denote the remaining element $[n+2 m] \backslash\{i, \pi(i)\}$. Let $\pi^{\prime}$ denote the permutation on the colored set $C_{\ell} \times\left\{1^{\prime}, 2^{\prime}, 3^{\prime}, \cdots,(m+n)^{\prime}\right\}$ such that $\operatorname{sign}_{\pi^{\prime}}\left(i^{\prime}\right)=1\left(i^{\prime} \in[m]\right)$ and $i^{\prime}\left(i^{\prime} \in[m]\right)$ belong into distinct cycles, by transforming the $i^{\prime}$ into $\{i, \pi(i)\}$, we obtain the desired permutation in $F_{\ell, n+m}^{m}$ and vice versa. From this construction, we have $f_{i x}(\pi)=f_{i x} x_{>m}\left(\pi^{\prime}\right)$. This completes the proof.

Theorem 46. For $\ell \geqslant 1$ and $0 \leqslant m \leqslant n$, we have

$$
\sum_{n \geqslant 0} f_{\ell, n}^{m}(\lambda) \frac{u^{n}}{n !}=\frac{u^{m} \exp (\lambda-1) u}{(1-\ell u)^{m+1}}
$$

Proof. According to the generating function (33) of $g_{\ell, n}^{m}(\lambda)$, it is clear to see that

$$
\sum_{n \geqslant 0} d_{\ell, n+m}^{m}(\lambda) \frac{u^{n}}{n !}=\frac{\exp (\lambda-1) u}{(1-\ell u)^{m+1}} .
$$

For the above identity, multiplying both sides by $u^{m}$, we obtain

$$
\sum_{n \geqslant 0} \frac{(n+m) !}{n !} d_{\ell, n+m}^{m}(\lambda) \frac{u^{n+m}}{(n+m) !}=\frac{u^{m} \exp (\lambda-1) u}{(1-\ell u)^{m+1}} .
$$

With Theorem 20,

$$
\sum_{n \geqslant 0} f_{\ell, n+m}^{m} \frac{u^{n+m}}{(n+m) !}=\frac{u^{m} \exp (\lambda-1) u}{(1-\ell u)^{m+1}},
$$

which is (49) by shifting $n+m$ to $n$.

With (3), (45), (46), (47) and Theorem 20, we obtain the following corollary.

Corollary 47. For $\ell \geqslant 1,1 \leqslant m \leqslant n-2$, we have

$$
\begin{aligned}
(n-m+1) f_{\ell, n}^{m-1}(\lambda) & =\ell m f_{\ell, n}^{m}(\lambda)+(\lambda-1) n f_{\ell, n-1}^{m-1}(\lambda) ; \\
(n-m) f_{\ell, n}^{m}(\lambda) & =n(\ell n-1+\lambda) f_{\ell, n-1}^{m}(\lambda)-\ell(\lambda-1) n(n-1) f_{\ell, n-2}^{m}(\lambda) ; \\
f_{\ell, n}^{m}(\lambda) & =\ell n f_{\ell, n-1}^{m}(\lambda)+n f_{\ell, n-1}^{m-1}(\lambda) ; \\
(n-m) f_{\ell, n}^{m}(\lambda) & =(\lambda-1) n(n-1) f_{\ell, n-2}^{m-1}(\lambda)+\ell n^{2} f_{\ell, n-1}^{m}(\lambda) ;
\end{aligned}
$$

where $f_{\ell, 0}^{0}(\lambda)=1, f_{\ell, 1}^{0}(\lambda)=\lambda+\ell-1$ and $f_{\ell, 1}^{1}(\lambda)=1$.

With (51) and (52), we have the following corollary.

Corollary 48. For $\ell \geqslant 1$ and $1 \leqslant m \leqslant n-2$, we have

$$
f_{\ell, n}^{m}(\lambda)=m f_{\ell, n-1}^{m-1}(\lambda)-\ell(\lambda-1)(n-1) f_{\ell, n-2}^{m}(\lambda)+(\ell m+\ell n-1+\lambda) f_{\ell, n-1}^{m}(\lambda) .
$$

Remark 49. When $(\ell, \lambda)=(1,0),(49)$ and (54) reduce to the results of $[14$, Theorem 3 and Theorem 2]. 


\section{Combinatorial proof of recurrence relation (46)}

In this section, we give the combinatorial proof of recurrence (46), that is,

$$
\sum_{\pi \in C_{\ell, n}^{m}} \lambda^{\mathrm{fix}>m(\pi)}=\ell(n-m) \sum_{\pi \in C_{\ell, n-1}^{m}} \lambda^{\mathrm{fix}>m(\pi)}+\sum_{\pi \in C_{\ell, n-1}^{m-1}} \lambda^{\mathrm{fix}>m-1}(\pi),
$$

other recurrences (45) and (47) can be proved in similar ways.

Lemma 50. For $0 \leqslant m \leqslant n$,

$$
\sum_{\substack{\pi \in C_{\ell, n}^{m} \\ m \in F I X(\pi)}} \lambda^{\mathrm{fix}>m(\pi)}=\sum_{\pi \in C_{\ell, n-1}^{m-1}} \lambda^{\mathrm{fix}>m-1}(\pi) .
$$

Proof. It follows similar arguments as in the proof of Lemma 21.

Lemma 51. For $0 \leqslant m \leqslant n$,

$$
\sum_{\substack{\pi \in C_{\ell, n}^{m} \\ m \notin F I X(\pi)}} \lambda^{\mathrm{fix}>m(\pi)}=\ell(n-m) \sum_{\pi \in C_{\ell, n-1}^{m}} \lambda^{\mathrm{fix}>m(\pi)} .
$$

Proof. Let us consider the map $\chi: \pi \rightarrow\left(\epsilon, \beta, \pi^{\prime}\right)$ from $C_{\ell, n}^{m} \cap\left\{\pi \in G_{\ell, n} \mid m \notin F I X(\pi)\right\}$ to $C_{\ell} \times[n-m] \times C_{\ell, n-1}^{m}$ such that $\operatorname{fix}_{>m}(\pi)=\operatorname{fix}_{>m}\left(\pi^{\prime}\right)$.

For $\pi \in C_{\ell, n}^{m} \cap\left\{\pi \in G_{\ell, n} \mid m \notin F I X(\pi)\right\}$, we decompose $\pi$ as the product of disjoint cycles. Let $\pi(m)=\beta$, it is easy to see $|\beta| \in[m+1, n]$ and $\operatorname{sign}_{\pi}(\beta)=\epsilon$.

For the element $i \in \pi$, we delete the element $\beta$ and define the element $i^{\prime} \in[n-1]$ in $\pi^{\prime}$ by

$$
i^{\prime}=\left\{\begin{array}{lll}
i, & \text { if } & |i|<|\beta| \\
i-1, & \text { if } & |i|>|\beta| .
\end{array}\right.
$$

Conversely, starting from $\left(\epsilon, \beta, \pi^{\prime}\right) \in C_{\ell} \times[n-k] \times C_{\ell, n-1}^{m}$, for the element $i^{\prime} \in \pi^{\prime}$, we define the element $i \in[n]$ in $\pi$ by

$$
i= \begin{cases}i^{\prime}, & \text { if } \quad\left|i^{\prime}\right|<|\beta| ; \\ i^{\prime}+1, & \text { if } \quad\left|i^{\prime}\right| \geqslant|\beta|,\end{cases}
$$

and let $\pi(m)=(\epsilon, \beta)$.

For example, let $\ell=4, n=9, k=4$, if $\pi=(1 \overline{\overline{7}})(2 \overline{\overline{5}})(3 \overline{\overline{8}})(4 \overline{9})(6) \in C_{4,9}^{4}$,

$$
\begin{aligned}
& \quad \epsilon=\zeta, \beta=9, \quad \pi^{\prime}=(1 \overline{\overline{\overline{7}}})(2 \overline{\overline{5}})(3 \overline{\overline{\overline{8}}})(4)(6) \in C_{4,8}^{4} \quad \text { and } \quad \operatorname{fix}_{>4}(\pi)=\mathrm{fix}_{>4}\left(\pi^{\prime}\right) . \\
& \text { for } \pi=(1 \overline{\overline{7}})(2)(3 \overline{8})(4 \overline{\overline{5}})(6)(9) \in C_{4,9}^{4}, \\
& \epsilon=\zeta^{2}, \beta=5, \quad \pi^{\prime}=(1 \overline{\overline{\overline{6}}})(2)(3 \overline{7})(4)(5)(8) \in C_{4,8}^{4} \quad \text { and } \quad \operatorname{fix}_{>4}(\pi)=\operatorname{fix}_{>4}\left(\pi^{\prime}\right) .
\end{aligned}
$$


Proof of equation (55). By considering the following equation,

$$
\sum_{\pi \in C_{\ell, n}^{m}} \lambda^{\mathrm{fix}>m(\pi)}=\sum_{\substack{\pi \in C_{\ell, n}^{m} \\ m \notin F I X(\pi)}} \lambda^{\mathrm{fix}>m(\pi)}+\sum_{\substack{\pi \in C_{\ell, n}^{m} \\ m \in F I X(\pi)}} \lambda^{\mathrm{fix}>m(\pi)},
$$

by Lemma 50 , the $(58)$ is equivalent to

$$
\sum_{\pi \in C_{\ell, n}^{m}} \lambda^{\mathrm{fix}>m(\pi)}=\sum_{\substack{\pi \in C_{\ell, n}^{m} \\ m \notin F I X(\pi)}} \lambda^{\mathrm{fix}>m(\pi)}+\sum_{\pi \in C_{\ell, n-1}^{m-1}} \lambda^{\mathrm{fix}>m-1}(\pi) .
$$

By Lemma 51, we obtain (55) immediately. This completes the proof.

\section{Final remarks}

Faliharimalala and Zeng [8, eq. (1.2)] studied the wreath product analogue of Euler's $q$-difference table $\left\{g_{\ell, n}^{m}(q)\right\}_{0 \leqslant m \leqslant n}$ as follows.

Definition 52 (Faliharimalala-Zeng). For fixed integer $\ell \geqslant 1$, the coefficients of Euler's $q$-difference table $\left(g_{\ell, n}^{m}(q)\right)_{0 \leqslant m \leqslant n}$ for $C_{\ell}$ 々 $S_{n}$ are defined by

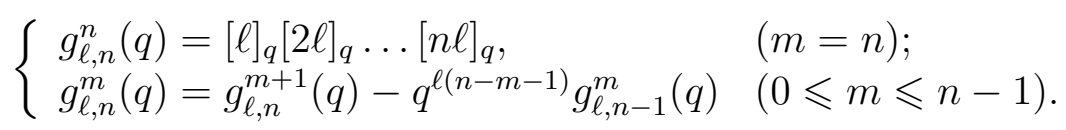

Faliharimalala and Zeng found a combinatorial interpretation of $\left(g_{\ell, n}^{m}(q)\right)_{0 \leqslant m \leqslant n}$ by introducing a new Mahonian statistic fmaf on the wreath products. So the natural question is to find a $q$ - $\lambda$-Euler's difference table for $\lambda$-Euler's difference table in Definition 1 , it seems the statistic fmaf cannot help directly.

\section{Acknowledgments}

I am grateful to my advisor Jiang Zeng for his guidance during this work. I also thank the referee for carefully reading the manuscript and giving constructive comments which helped improve the paper.

\section{References}

[1] K. S. Briggs, J. B. Remmel, $m$-Rook numbers and a generalization of a formula of Frobenius to $C_{m} 2 S_{n}$, J. Combin. Theory Ser. A, 113 (2006) 1138-1171.

[2] W. Y. C. Chen, C. C. Y. Gu, K. J. Ma, L. X. W. Wang, Higher order log-concavity in Euler's difference table, Discrete Math. 311 (2011), no. 20, 2128-2134.

[3] R. J. Clarke, G. N. Han, J. Zeng, A combinatorial interpretation of the Seidel generation of $q$-derangement numbers, Ann. Comb. 1(1997) 313-327. 
[4] D. Dumont, A. Randrianarivony, Dérangements et nombres de Genocchi, Discrete Math. 132 (1994) 37-49.

[5] N. Eriksen, R. Freij, J. Wästlund, Enumeration of derangements with descents in prescribed positions, Electron. J. Combin., 16(1) (2009) \#R32.

[6] H. L. M. Faliharimalala, Combinatorial studies of Euler's table on wreath products, Thèse de doctorat, Université Claude Bernard Lyon 1, 2010.

[7] H. L. M. Faliharimalala, J. Zeng, Derangements and Euler's difference table for $C_{l} S_{n}$, Electron. J. Combin., 15 (2008) \#R65.

[8] H. L. M. Faliharimalala, J. Zeng, Fix-Euler-Mahonian statistics on wreath products, Adv. Appl. Math., 46 (2011), 275-295.

[9] D. Foata, M. P. Schützenberger, Théorie géométrique des polynômes eulériens, Lecture Notes in Math., vol. 138, Springer-Verlag, Berlin, 1970.

[10] J. Liese, J. B. Remmel, $Q$-analogues of the number of permutations with $k$ excedances, Pure Mathematics and Applications, 21 (2010), 285-320.

[11] F. Rakotondrajao, On Euler's difference table, Proc. of FPSAC'07, Tianjin, 2007.

[12] F. Rakotondrajao, $k$-fixed-points-permutations, Integers 7 (2007) \#A36.

[13] R. P. Stanley, Enumerative Combinatorics, vol. 1, Cambridge University Press, Cambridge, 1997.

[14] C. Y. Wang, P. Miska, I. Mezö, The r-derangement numbers. Discrete Math. 340 (2017), no. 7, 1681-1692. 